\title{
Diffeomorphisms with weak shadowing
}

\author{
by
}

\author{
Kazuhiro Sakai (Yokohama)
}

\begin{abstract}
The weak shadowing property is really weaker than the shadowing property. It is proved that every element of the $C^{1}$ interior of the set of all diffeomorphisms on a $C^{\infty}$ closed surface having the weak shadowing property satisfies Axiom A and the no-cycle condition (this result does not generalize to higher dimensions), and that the non-wandering set of a diffeomorphism $f$ belonging to the $C^{1}$ interior is finite if and only if $f$ is Morse-Smale.
\end{abstract}

The notion of pseudo-orbits very often appears in several branches of the modern theory of dynamical systems; especially, the shadowing property usually plays an important role in stability theory. The weak shadowing property (which is really weaker than the shadowing property) was introduced in [6]. Corless and Pilyugin (see [6, p. 30]) proved that the weak shadowing property is generic in the set of all homeomorphisms on a $C^{\infty}$ closed manifold endowed with $C^{0}$ topology (see [7] for further results). Every diffeomorphism having the shadowing property has the weak shadowing property but the converse is not true. Indeed, an irrational rotation on the unit circle has the weak shadowing property but does not have the shadowing property.

The purpose of this paper is to investigate the dynamics of a diffeomorphism belonging to the $C^{1}$ interior of the set of all diffeomorphisms on a $C^{\infty}$ closed surface with the weak shadowing property. The motivation is the following remarkable example on the 2-torus constructed by Plamenevskaya [8] (see also [7]). We describe it briefly before stating our results.

ExAmple $([8])$. There exists a diffeomorphism $g$ on the 2-torus $\mathbb{T}^{2}$ satisfying Axiom A and the no-cycle condition such that $g$ has the weak shad-

2000 Mathematics Subject Classification: 37B99, 37C50, 37C75, 37D15, 37D20.

Key words and phrases: pseudo-orbits, weak shadowing property, shadowing property, Morse-Smale, Axiom A, no-cycle condition, strong transversality condition, structurally stable. 
owing property but does not have the shadowing property. More precisely, represent $\mathbb{T}^{2}$ as the square $[-2,2] \times[-2,2]$ with opposite sides identified. Consider the metric $d$ generated by the usual metric on $\mathbb{R}^{2}$. Let $g: \mathbb{T}^{2} \rightarrow \mathbb{T}^{2}$ be a diffeomorphism with the following properties:

(1) the non-wandering set $\Omega(g)$ consists of 4 hyperbolic fixed points; that is, $\Omega(g)=\left\{p_{1}, p_{2}, p_{3}, p_{4}\right\}$, where $p_{1}$ is a sink, $p_{4}$ is a source and $p_{2}, p_{3}$ are saddles,

(2) in the coordinates $(v, w) \in[-2,2] \times[-2,2]$, the following conditions hold:

(2.1) $p_{1}=(1,2), p_{2}=(-1,0), p_{3}=(1,0), p_{4}=(-1,2)$,

(2.2) $W^{\mathrm{s}}\left(p_{2}\right) \cup\left\{p_{3}\right\}=W^{\mathrm{u}}\left(p_{3}\right) \cup\left\{p_{2}\right\}=[-2,2] \times\{0\}$,

$$
W^{\mathrm{u}}\left(p_{2}\right)=\{-1\} \times(-2,2), \quad W^{\mathrm{s}}\left(p_{3}\right)=\{1\} \times(-2,2),
$$

where $W^{\mathrm{s}}\left(p_{i}\right)$ and $W^{\mathrm{u}}\left(p_{i}\right)$ are the stable and unstable manifolds respectively for $i=2,3$,

(2.3) there exist neighborhoods $U_{2}, U_{3}$ of $p_{2}, p_{3}$ such that

$$
g(x)=p_{i}+D_{p_{i}} g\left(x-p_{i}\right) \quad \text { if } x \in U_{i} \text { for } i=2,3,
$$

(2.4) there exists a neighborhood $U$ of $z=(0,0)$ such that $g(U) \subset$ $U_{2}, g^{-1}(U) \subset U_{3}$ and $g^{-1}$ is affine on $g(U)$,

(2.5) the eigenvalues of $D_{p_{2}} g$ are $-\mu, \nu$ with $\mu>1,0<\nu<1$, and the eigenvalues of $D_{p_{3}} g$ are $-\lambda, \kappa$ with $0<\lambda<1, \kappa>1$.

It is proved in [8] that $g$ has the weak shadowing property if and only if the number $\log \lambda / \log \mu$ is irrational. Note that $g$ does not have the shadowing property since $g$ does not satisfy the $C^{0}$ transversality condition (see [7] and [11]).

Let $M$ be a $C^{\infty}$ closed manifold and let $\operatorname{Diff}(M)$ be the space of $C^{1}$ diffeomorphisms on $M$ endowed with the $C^{1}$ topology. Denote by $\mathcal{W S}(M)$ the set of all $f \in \operatorname{Diff}(M)$ having the weak shadowing property. In this paper, the following is proved.

Theorem 1. Let $M$ be a $C^{\infty}$ closed surface. Then every element of the $C^{1}$ interior of $\mathcal{W S}(M)$, int $\mathcal{W S}(M)$, satisfies Axiom $A$ and the no-cycle condition.

The theorem does not generalize to higher dimensions. Actually, it is proved in [5] that there is a $C^{1}$ (non-empty) open set $\mathcal{T} \subset \operatorname{Diff}\left(\mathbb{T}^{3}\right)$ such that every $g \in \mathcal{T}$ is topologically transitive but not Anosov (see also [1] for a generalization). It is easy to see that every $g \in \mathcal{T}$ has the weak shadowing property but does not satisfy Axiom A (and the no-cycle condition).

Recall that $f \in \operatorname{Diff}(M)$ is called Morse-Smale if (a) the non-wandering set of $f$ is a finite union of hyperbolic periodic points, (b) the stable and 
unstable manifolds of the periodic points are all transversal. Every MorseSmale diffeomorphism $f$ has the weak shadowing property since $f$ has the shadowing property (cf. [9]). Hence, the structural stability of $f$ implies that $f \in \operatorname{int} \mathcal{W S}(M)$. In this paper, the following is also proved.

Theorem 2. Let $M$ be a $C^{\infty}$ closed surface. Then the non-wandering set of $f \in \operatorname{int} \mathcal{W S}(M)$ is finite if and only if $f$ is Morse-Smale.

By Theorem 2, Plamenevskaya's map (in the case of $\log \lambda / \log \mu$ irrational) belongs to the boundary of $\mathcal{W S}\left(\mathbb{T}^{2}\right)$.

Let $M$ be as before and denote by $\mathcal{S}(M)$ the set of all $f \in \operatorname{Diff}(M)$ having the shadowing property. Then $\mathcal{S}(M) \subset \mathcal{W S}(M)$. The $C^{1}$ interior of $\mathcal{S}(M)$, int $\mathcal{S}(M)$, was characterized as the set of all diffeomorphisms satisfying Axiom A and the strong transversality condition; that is, int $\mathcal{S}(M)$ coincides with the set of all structurally stable diffeomorphisms (see [7], [9] and [10]). Therefore, once Theorem 2 is established, we have the following

Corollary. Let $M$ be a $C^{\infty}$ closed surface, and let $f \in \operatorname{Diff}(M)$. If $\Omega(f)$ is finite, then the following conditions are equivalent:

(i) $f \in \operatorname{int} \mathcal{W S}(M)$,

(ii) $f \in \operatorname{int} \mathcal{S}(M)$.

Let $M$ be as before, and let $P(f)$ be the set of all periodic points of $f \in \operatorname{Diff}(M)$. Then $P(f)$ is a subset of the non-wandering set $\Omega(f)$. We denote by $\mathcal{F}(M)$ the set of all $f$ having a $C^{1}$ neighborhood $\mathcal{U}(f) \subset \operatorname{Diff}(M)$ such that every $p \in P(g)(g \in \mathcal{U}(f))$ is hyperbolic. Then $\mathcal{F}(M)$ can be characterized as the set of all diffeomorphisms satisfying Axiom $\mathrm{A}$ and the no-cycle condition (see [3]). We denote by $\mathcal{A N}(M)$ the set of all $f$ satisfying Axiom $\mathrm{A}$ and the no-cycle condition.

Hereafter, let $M$ be a $C^{\infty}$ closed surface. Our theorems follow from the next two propositions.

Proposition A. int $\mathcal{W S}(M) \subset \mathcal{F}(M)$.

Proposition B. Suppose $f \in \mathcal{A N}(M)$, and $\Omega(f)$ is finite. If $f \in$ $\operatorname{int} \mathcal{W S}(M)$, then the stable and unstable manifolds are all transversal.

In the proof of Proposition B, on the assumption that $f \in \operatorname{int} \mathcal{W S}(M)$ does not satisfy the strong transversality condition, we construct a new diffeomorphism $g\left(C^{1}\right.$-near $\left.f\right)$ with the same geometric structure as Plamenevskaya's map linearizing $f$ at suitable points with a small perturbation. By construction, we can show that $g$ does not have the weak shadowing property. But this is a contradiction since the perturbed map $g$ is $C^{1}$-near $f$.

REMARK. (i) By Theorem 1, int $\mathcal{W S}(M) \subset \mathcal{A N}(M)$, but the converse inclusion does not hold. Indeed, let $g: \mathbb{T}^{2} \rightarrow \mathbb{T}^{2}$ be Plamenevskaya's map. Then it can be easily checked that $g^{2} \in \mathcal{A N}\left(\mathbb{T}^{2}\right)$, but $g^{2}$ does not have the 
weak shadowing property (since all eigenvalues of the saddles are positive with respect to $g^{2}$ ).

(ii) If $f \in \operatorname{Diff}(M)$ has the shadowing property, then so does $f^{n}$ for all $n \in \mathbb{Z}$. However, this is not true for the weak shadowing property. Indeed, Plamenevskaya's map $g: \mathbb{T}^{2} \rightarrow \mathbb{T}^{2}$ has the weak shadowing property (for $\log \lambda / \log \mu$ irrational), but as stated above, $g^{2}$ does not have the weak shadowing property.

1. Preliminaries and proof of Proposition A. Let $d$ be the distance on $M$ induced by a Riemannian metric on $T M$, and let $f \in \operatorname{Diff}(M)$. Denote by $\mathbf{O}_{f}(x)$ the $f$-orbit $\left\{f^{i}(x)\right\}_{i \in \mathbb{Z}}$ of $x \in M$. A sequence $\left\{x_{i}\right\}_{i \in \mathbb{Z}} \subset M$ is called a $\delta$-pseudo-orbit of $f$ if $d\left(f\left(x_{i}\right), x_{i+1}\right)<\delta$ for $i \in \mathbb{Z}$. Given $\varepsilon>0,\left\{x_{i}\right\}_{i \in \mathbb{Z}}$ is said to be $\varepsilon$-shadowed by $y \in M$ if $d\left(f^{i}(y), x_{i}\right)<\varepsilon$ for $i \in \mathbb{Z}$. We say that $f$ has the shadowing property if for every $\varepsilon>0$, there exists $\delta>0$ such that every $\delta$-pseudo-orbit of $f$ can be $\varepsilon$-shadowed by some point.

Given $\varepsilon>0,\left\{x_{i}\right\}_{i \in \mathbb{Z}}$ is said to be weakly $\varepsilon$-shadowed by $y \in M$ if $\left\{x_{i}\right\}_{i \in \mathbb{Z}} \subset U_{\varepsilon}\left(\mathbf{O}_{f}(y)\right)$. Here $U_{\varepsilon}(A)=\{y \in M$ : there is $x \in A$ such that $d(x, y)<\varepsilon\}$ is the $\varepsilon$-neighborhood of a subset $A$ of $M$. We say that $f$ has the weak shadowing property if for every $\varepsilon>0$, there exists $\delta>0$ such that every $\delta$-pseudo-orbit of $f$ can be weakly $\varepsilon$-shadowed by some point. As stated before, it is easy to see that if $f$ has the shadowing property (or if $f$ is topologically transitive), then $f$ has the weak shadowing property.

Since $M$ is compact, there are a family $\left\{\left(U_{i}, \varphi_{i}\right)\right\}_{i=1}^{I}$ of local charts and a constant $\alpha>0$ such that for all $x \in M$, there exists $\left(U_{i}, \varphi_{i}\right)$ with $U_{\alpha}(x) \subset U_{i}$. Take $0<\beta<\alpha$ small enough so that for all $x \in M$, there are $\left(U_{i}, \varphi_{i}\right)$ and $\left(U_{j}, \varphi_{j}\right)$ satisfying $U_{\beta}(x) \subset U_{i}$ and $f\left(U_{\beta}(x)\right) \subset U_{\alpha}(f(x)) \subset U_{j}$. For convenience, denote $\varphi_{i}$ by $\varphi_{x}$ and $\varphi_{j}$ by $\varphi_{f(x)}$ respectively (sometimes $\varphi_{x}$ will be denoted by $\varphi$ if no confusion can arise).

Let $B_{\varepsilon}(x)=\{y \in M: d(x, y) \leq \varepsilon\}$ for $x \in M$ and $\varepsilon>0$, and let $|\cdot|=|\cdot|_{\mathbb{R}^{2}}$ be the usual metric on $\mathbb{R}^{2}$. Put

$$
F_{x}=\varphi_{f(x)} \circ f \circ \varphi_{x}^{-1}: \varphi_{x}\left(U_{\beta}(x)\right) \rightarrow \mathbb{R}^{2} \quad(x \in M) .
$$

Then the following is established by mimicking the proof of [2, Lemma 1.1].

Lemma 1.1. Let $\mathcal{U}(f)$ be a $C^{1}$ neighborhood of $f$, and let $f^{n}(p)=p$ $(n \geq 1)$ be given. Then there are $0<\varepsilon_{0}<\beta / 4$ and $\delta_{0}>0$ such that for every linear isomorphism $\mathcal{O}: \mathbb{R}^{2} \rightarrow \mathbb{R}^{2}$ with $\|\mathcal{O}-I\|<\delta_{0}$, there exists $g \in \mathcal{U}(f)$ satisfying

(i) $B_{4 \varepsilon_{0}}\left(f^{i}(p)\right) \cap B_{4 \varepsilon_{0}}\left(f^{j}(p)\right)=\emptyset$ for $0 \leq i \neq j \leq n-1$,

(ii) $g(x)=f(x)$ if $x \in\left\{p, f(p), \ldots, f^{n-1}(p)\right\} \cup\left\{M \backslash \bigcup_{i=0}^{n-1} B_{4 \varepsilon_{0}}\left(f^{i}(p)\right)\right\}$,

(iii) $g(x)=\varphi^{-1}\left(\varphi\left(f^{i+1}(p)\right)+D_{\varphi\left(f^{i}(p)\right)} F_{f^{i}(p)}\left(\varphi(x)-\varphi\left(f^{i}(p)\right)\right)\right)$ if $x \in$ $B_{\varepsilon_{0}}\left(f^{i}(p)\right)$ for $0 \leq i \leq n-2$, 
(iv) $g(x)=\varphi^{-1}\left(\varphi\left(f^{n}(p)\right)+\mathcal{O} \circ D_{\varphi\left(f^{n-1}(p)\right)} F_{f^{n-1}(p)}\left(\varphi(x)-\varphi\left(f^{n-1}(p)\right)\right)\right)$ if $x \in B_{\varepsilon_{0}}\left(f^{n-1}(p)\right)$.

Here $I: \mathbb{R}^{2} \rightarrow \mathbb{R}^{2}$ is the identity map and $\|\cdot\|$ is the norm induced by $|\cdot| \cdot$

Define

$$
L(\mathbf{v})=\left\{\begin{array}{c}
\varphi\left(f^{i+1}(p)\right)+D_{\varphi\left(f^{i}(p)\right)} F_{f^{i}(p)}\left(\mathbf{v}-\varphi\left(f^{i}(p)\right)\right) \\
\text { if } \mathbf{v} \in \varphi\left(B_{\varepsilon_{0}}\left(f^{i}(p)\right)\right) \text { for } 0 \leq i \leq n-2, \\
\varphi\left(f^{n}(p)\right)+\mathcal{O} \circ D_{\varphi\left(f^{n-1}(p)\right)} F_{f^{n-1}(p)}\left(\mathbf{v}-\varphi\left(f^{n-1}(p)\right)\right) \\
\text { if } \mathbf{v} \in \varphi\left(B_{\varepsilon_{0}}\left(f^{n-1}(p)\right)\right) .
\end{array}\right.
$$

If we take $0<\varepsilon_{1}<\varepsilon_{0}$ so small that $g^{i}\left(B_{\varepsilon_{1}}(p)\right) \subset B_{\varepsilon_{0}}\left(g^{i}(p)\right)$ for $1 \leq i \leq n$, then $L^{n}: \varphi\left(B_{\varepsilon_{1}}(p)\right) \rightarrow \mathbb{R}^{2}$ is well defined.

REMARK. The map $L^{n}{ }_{\mid \varphi\left(B_{\varepsilon_{1}}(p)\right)}$ is conjugate to $g^{n}{ }_{\mid B_{\varepsilon_{1}}(p)}$ by $\varphi$. In the proof of Proposition A, we mainly consider $L$ instead of $g$ for simplicity, because the argument is almost local.

Proof of Proposition A. Let $f \in \operatorname{int} \mathcal{W S}(M)$. It is enough to show that every $f^{n}(p)=p \in P(f)(n \geq 1)$ is hyperbolic since int $\mathcal{W} \mathcal{S}(M)$ is an open set. Assuming that there is a non-hyperbolic periodic point $f^{n}(p)=p$, we shall derive a contradiction. To simplify notation, we consider the case $n=1$ (although the other case is treated similarly, a rough outline of the proof will be given later on).

Fix a $C^{1}$ neighborhood $\mathcal{U}(f) \subset \mathcal{W} \mathcal{S}(M)$ and let

$$
F_{p}=\varphi_{p} \circ f \circ \varphi_{p}^{-1}: \varphi_{p}\left(U_{\beta}(p)\right) \rightarrow \mathbb{R}^{2} .
$$

We may suppose that $\varphi(p)$ coincides with the origin $O$ of $\mathbb{R}^{2}$. Clearly, $D_{O} F_{p}$ is not hyperbolic.

The proof is divided into two cases.

CASE 1: The eigenvalues of $D_{O} F_{p}$ are real. Applying Lemma 1.1 to $f(p)=p$ we have the following

Claim 1.1. Let $\varepsilon_{0}, \delta_{0}>0$ be given by Lemma 1.1 for the above $\mathcal{U}(f)$ and $p$. Then there exists a linear isomorphism $\mathcal{O}: \mathbb{R}^{2} \rightarrow \mathbb{R}^{2}$ with $\|\mathcal{O}-I\|<\delta_{0}$ such that for the diffeomorphism $g \in \mathcal{U}(f)(g(p)=p)$ given by Lemma 1.1 for the $\mathcal{O}$, we have the following;

(i) there exists $0<\varepsilon_{1}<\varepsilon_{0}$ such that $B_{\varepsilon_{1}}(p) \subset g\left(B_{\varepsilon_{0}}(p)\right) \cap g^{-1}\left(B_{\varepsilon_{0}}(p)\right)$ and $g_{\mid B_{\varepsilon_{1}}(p)}\left(\right.$ resp. $\left.g_{\mid B_{\varepsilon_{1}}(p)}^{-1}\right)$ is conjugate to

$$
L=\mathcal{O} \circ D_{O} F_{p \mid \varphi\left(B_{\varepsilon_{1}}(p)\right)} \quad\left(\text { resp. } L^{-1}=\left(\mathcal{O} \circ D_{O} F_{p}\right)_{\mid \varphi\left(B_{\varepsilon_{1}}(p)\right)}^{-1}\right),
$$

(ii) if we extend $L$ to a linear isomorphism on $\mathbb{R}^{2}$, then there are constants $\lambda, \mu \in \mathbb{R}(|\lambda|=1,|\mu| \neq 1)$ and an L-invariant splitting $E^{1} \oplus E^{2}\left(\operatorname{dim} E^{i}=1\right.$ for $i=1,2)$ such that $L(\mathbf{v})=\lambda \mathbf{v}$ for $\mathbf{v} \in E^{1}$ and $L(\mathbf{v})=\mu \mathbf{v}$ for $\mathbf{v} \in E^{2}$. 
Proof. See the proof of [12, Lemma 2].

Let $E^{1}$ and $E^{2}$ be as in Claim 1.1(ii). For $\varepsilon>0$, put $E_{\varepsilon}^{i}=\left\{\mathbf{v} \in E^{i}\right.$ : $|\mathbf{v}| \leq \varepsilon\}(i=1,2)$ and

$$
\mathcal{B}_{\varepsilon}(O)=E_{\varepsilon}^{1} \times E_{\varepsilon}^{2} .
$$

Then $\mathcal{B}_{\varepsilon}(O)$ is a closed neighborhood of the origin. Take $0<\varepsilon_{2}<\varepsilon_{1}$ so small that $\mathcal{B}_{\varepsilon_{2}}(O) \subset \varphi\left(B_{\varepsilon_{1}}(p)\right)$. If a point $\mathbf{v} \in \mathbb{R}^{2}$ has coordinates $(v, w)$ with respect to $E^{1} \times E^{2}$, then

$$
L(v, w)=(\lambda v, \mu w), \quad(v, w) \in \mathcal{B}_{\varepsilon_{2}}(O) .
$$

Note that

$$
\varphi \circ g \circ \varphi^{-1}(\mathbf{v})=L(\mathbf{v}) \text { and } \varphi \circ g^{-1} \circ \varphi^{-1}(\mathbf{v})=L^{-1}(\mathbf{v})
$$

while $\mathbf{v} \in \mathcal{B}_{\varepsilon_{2}}(O)$ by Claim 1.1(i).

Since $g$ has the weak shadowing property, we have the following

Claim 1.2. For every $0<\varepsilon<\varepsilon_{2} / 2$, there is $0<\delta=\delta(\varepsilon)<\varepsilon$ such that for any $\delta$-pseudo-orbit $\left\{\mathbf{x}_{k}\right\}_{k \in \mathbb{Z}} \subset \mathcal{B}_{\varepsilon_{2} / 2}(O)$ of $L$ (that is, $\left|L\left(\mathbf{x}_{k}\right)-\mathbf{x}_{k+1}\right|<\delta$ for $k \in \mathbb{Z})$, we can find $\mathbf{z} \in \mathcal{B}_{\varepsilon}\left(\mathbf{x}_{0}\right)$ such that $\left\{\mathbf{x}_{k}\right\}_{k \in \mathbb{Z}}$ is contained in the $\varepsilon$-neighborhood of

$$
\varphi\left(\mathbf{O}_{g}\left(\varphi^{-1}(\mathbf{z})\right) \cap \varphi^{-1}\left(\mathcal{B}_{\varepsilon_{2}}(O)\right)\right) .
$$

Take $\mathbf{v}_{1} \in E_{\varepsilon_{2}}^{1}$ with $\left|\mathbf{v}_{1}\right|=\varepsilon_{2} / 2$, and put

$$
F^{+}=E_{\varepsilon_{2}}^{2}+\mathbf{v}_{1} / 2 \text { and } F^{-}=E_{\varepsilon_{2}}^{2}-\mathbf{v}_{1} / 2 .
$$

Let $d_{1}=\inf \left\{|\mathbf{w}|: \mathbf{w} \in F^{+} \cup F^{-}\right\}, d_{2}=\inf \left\{\left|\mathbf{v}_{1}-\mathbf{w}\right|: \mathbf{w} \in F^{+} \cup F^{-}\right\}$and $d_{3}=\inf \left\{\left|\mathbf{v}_{1}+\mathbf{w}\right|: \mathbf{w} \in F^{+} \cup F^{-}\right\}$. Fix $0<\varepsilon<\min \left\{\varepsilon_{2} / 5, d_{1}, d_{2}, d_{3}\right\}$ and let $0<\delta=\delta(\varepsilon)<\varepsilon$ be given by Claim 1.2 .

Let $\mathcal{I}=\left\{t \mathbf{v}_{1}: t \in[-1,1]\right\} \subset E_{\varepsilon_{2} / 2}^{1}$ be an arc, and take a finite sequence $\left\{\mathbf{w}_{k}\right\}_{k=0}^{K} \subset \mathcal{I}$ (for some $K>0$ ) such that $\mathbf{w}_{0}=O, \mathbf{w}_{K}=\mathbf{v}_{1}\left(\right.$ or $\left.-\mathbf{v}_{1}\right)$ and

$$
\left|L\left(\mathbf{w}_{k}\right)-\mathbf{w}_{k+1}\right|<\delta \quad \text { for } 0 \leq k \leq K-1,
$$

where the $\mathbf{w}_{k}$ are chosen so that if $\mathbf{w}_{k}=t_{k} \mathbf{v}_{1}$, then $\left|t_{k}\right|<\left|t_{k+1}\right|$ for $0 \leq$ $k \leq K-1$. Put

$$
\begin{cases}\mathbf{x}_{k}=\mathbf{w}_{0} & \text { for } k \leq 0, \\ \mathbf{x}_{k}=\mathbf{w}_{k} & \text { for } 0 \leq k \leq K-1, \\ \mathbf{x}_{k}=L^{k-K}\left(\mathbf{w}_{K}\right) & \text { for } k \geq K .\end{cases}
$$

Then $\left\{\mathbf{x}_{k}\right\}_{k \in \mathbb{Z}}$ is a $\delta$-pseudo-orbit of $L$ in $\mathcal{B}_{\varepsilon_{2} / 2}(O)$.

By Claim 1.2, there exists $\mathbf{z} \in \mathcal{B}_{\varepsilon}(O)$ such that $\left\{\mathbf{x}_{k}\right\}_{k \in \mathbb{Z}}$ is contained in the $\varepsilon$-neighborhood of $\varphi\left(\mathbf{O}_{g}\left(\varphi^{-1}(\mathbf{z})\right) \cap \varphi^{-1}\left(\mathcal{B}_{\varepsilon_{2}}(O)\right)\right)$. We have $\mathbf{z} \notin E_{\varepsilon_{2}}^{1}$. Indeed, if $\mathbf{z} \in E_{\varepsilon_{2}}^{1}$, then $\mathbf{z} \in E_{\varepsilon}^{1}$, and thus

$$
\left|\varphi \circ g^{k} \circ \varphi^{-1}(\mathbf{z})-\mathbf{x}_{K}\right|=\left|L^{k}(\mathbf{z})-\mathbf{x}_{K}\right|>\varepsilon
$$

for all $k \in \mathbb{Z}$ by formulae (2), (13) and the choice of $\varepsilon$. This is a contradiction. 
If $|\mu|>1$, then, by (2) we can find $k^{\prime} \geq 0$ such that $L^{k}(\mathbf{z}) \in \mathcal{B}_{\varepsilon_{2}}(O)$ for $0 \leq k \leq k^{\prime}-1$ and $L^{k^{\prime}}(\mathbf{z}) \notin \mathcal{B}_{\varepsilon_{2}}(O)$ (because $\left.\mathbf{z} \notin E_{\varepsilon_{2}}^{1}\right)$. Thus $g^{k}\left(\varphi^{-1}(\mathbf{z})\right) \in$ $\varphi^{-1}\left(\mathcal{B}_{\varepsilon_{2}}(O)\right)$ for $0 \leq k \leq k^{\prime}-1$ and $g^{k^{\prime}}\left(\varphi^{-1}(\mathbf{z})\right) \notin \varphi^{-1}\left(\mathcal{B}_{\varepsilon_{2}}(O)\right)$. Let us prove $g^{k}\left(\varphi^{-1}(\mathbf{z})\right) \notin \varphi^{-1}\left(\mathcal{B}_{\varepsilon_{2}}(O)\right)$ for all $k \geq k^{\prime}$. Assume that there exists $k^{\prime \prime}>k^{\prime}$ such that $g^{k}\left(\varphi^{-1}(\mathbf{z})\right) \notin \varphi^{-1}\left(\mathcal{B}_{\varepsilon_{2}}(O)\right)$ for $k^{\prime} \leq k \leq k^{\prime \prime}-1$ and $g^{k^{\prime \prime}}\left(\varphi^{-1}(\mathbf{z})\right) \in \varphi^{-1}\left(\mathcal{B}_{\varepsilon_{2}}(O)\right)$. Since $L^{-k} \circ \varphi\left(g^{k^{\prime \prime}}\left(\varphi^{-1}(\mathbf{z})\right)\right) \in \mathcal{B}_{\varepsilon_{2}}(O)$ for all $k \geq 0$ by (2) and (3), we conclude that

$$
\begin{aligned}
L^{k^{\prime}-k^{\prime \prime}} \circ \varphi\left(g^{k^{\prime \prime}}\left(\varphi^{-1}(\mathbf{z})\right)\right) & =\varphi \circ g^{k^{\prime}-k^{\prime \prime}}\left(g^{k^{\prime \prime}}\left(\varphi^{-1}(\mathbf{z})\right)\right) \\
& =\varphi\left(g^{k^{\prime}}\left(\varphi^{-1}(\mathbf{z})\right)\right) \in \mathcal{B}_{\varepsilon_{2}}(O) .
\end{aligned}
$$

Thus $g^{k^{\prime}}\left(\varphi^{-1}(\mathbf{z})\right) \in \varphi^{-1}\left(\mathcal{B}_{\varepsilon_{2}}(O)\right)$. This is a contradiction. Hence, $g^{k}\left(\varphi^{-1}(\mathbf{z})\right)$ $\notin \varphi^{-1}\left(\mathcal{B}_{\varepsilon_{2}}(O)\right)$ for all $k \geq k^{\prime}$ and so

$$
\varphi\left(\left\{g^{k}\left(\varphi^{-1}(\mathbf{z})\right)\right\}_{k \geq k^{\prime}} \cap \varphi^{-1}\left(\mathcal{B}_{\varepsilon_{2}}(O)\right)\right)=\emptyset .
$$

Therefore $\left\{\mathbf{x}_{k}\right\}_{k \in \mathbb{Z}}$ must be contained in the $\varepsilon$-neighborhood of $\left\{L^{k}(\mathbf{z})\right\}_{k \leq k^{\prime}-1}$ in $\mathcal{B}_{\varepsilon_{2}}(O)$ by (3). But $\left|L^{k}(\mathbf{z})-\mathbf{x}_{K}\right|>\varepsilon$ for $0 \leq k \leq k^{\prime}-1$ by the choice of $\varepsilon$. Moreover, $\left|L^{-k}(\mathbf{z})-\mathbf{x}_{K}\right|>\varepsilon$ for all $k \geq 0$ by (2). This is a contradiction.

If $|\mu|<1$, then we arrive at a contradiction in the same way. Thus $p$ is hyperbolic.

CASE 2: The eigenvalues of $D_{O} F_{p}$ are complex. $\operatorname{Recall} \mathcal{U}(f) \subset \mathcal{W S}(M)$. In this case, by Lemma 1.1 we construct $g\left(C^{1}\right.$-near $\left.f\right)$ with a periodic point $q=g^{l}(q)$ (for some $l>0$ ) near $p$ such that the restriction of $g^{l}$ to a neighborhood of $q$ is conjugate to the (locally defined) identity map.

Claim 2.1. Let $\varepsilon_{0}, \delta_{0}>0$ be as in Lemma 1.1 for the above $\mathcal{U}(f)$ and $p$. Then there exists a linear isomorphism $\mathcal{O}: \mathbb{R}^{2} \rightarrow \mathbb{R}^{2}$ with $\|\mathcal{O}-I\|<\delta_{0}$ such that for the diffeomorphism $g \in \mathcal{U}(f)(g(p)=p)$ given by Lemma 1.1 for the $\mathcal{O}$, we have the following:

(i) there exists $0<\varepsilon_{1}<\varepsilon_{0}$ such that $B_{\varepsilon_{1}}(p) \subset g\left(B_{\varepsilon_{0}}(p)\right) \cap g^{-1}\left(B_{\varepsilon_{0}}(p)\right)$ and $g_{\mid B_{\varepsilon_{1}}(p)}\left(\right.$ resp. $g_{\mid B_{\varepsilon_{1}}(p)}^{-1}$ ) is conjugate to

$$
L=\mathcal{O} \circ D_{O} F_{p \mid \varphi\left(B_{\varepsilon_{1}}(p)\right)} \quad\left(\text { resp. } L^{-1}=\left(\mathcal{O} \circ D_{O} F_{p}\right)_{\mid \varphi\left(B_{\varepsilon_{1}}(p)\right)}^{-1}\right),
$$

(ii) if we extend $L$ to a linear isomorphism on $\mathbb{R}^{2}$, then there exists a (minimal) $l>0$ such that $L^{l}(\mathbf{v})=\mathbf{v}$ for all $\mathbf{v} \in \mathbb{R}^{2}$.

Proof. See the proof of [12, Lemma 2].

Let $\mathcal{B}_{\varepsilon}(\mathbf{v})=\left\{\mathbf{w} \in \mathbb{R}^{2}:|\mathbf{v}-\mathbf{w}| \leq \varepsilon\right\}$ for $\varepsilon>0$ and $\mathbf{v} \in \mathbb{R}^{2}$, and fix $0<$ $\varepsilon_{2}<\varepsilon_{1}$ such that $B_{\varepsilon_{2}}(p) \subset g^{-i}\left(B_{\varepsilon_{1}}(p)\right) \cap g^{i}\left(B_{\varepsilon_{1}}(p)\right)$ for $1 \leq i \leq l-1$. Thus, by Claim 2.1, if we choose $0<\varepsilon_{3}<\varepsilon_{2}$ so small that $\mathcal{B}_{\varepsilon_{3}}(O) \subset \varphi\left(B_{\varepsilon_{2}}(p)\right)$, 
then

$$
\varphi \circ g^{i} \circ \varphi^{-1}(\mathbf{v})=L^{i}(\mathbf{v}) \text { and } \varphi \circ g^{-i} \circ \varphi^{-1}(\mathbf{v})=L^{-i}(\mathbf{v})
$$

for $1 \leq i \leq l$ and

$$
L^{l}(\mathbf{v})=\mathbf{v}
$$

for $\mathbf{v} \in \mathcal{B}_{\varepsilon_{3}}(O)$.

Since $g$ has the weak shadowing property, we have the following

Claim 2.2. For every $0<\varepsilon<\varepsilon_{3} / 2$, there exists $0<\delta=\delta(\varepsilon)<\varepsilon$ such that for any $\delta$-pseudo-orbit $\left\{\mathbf{x}_{k}\right\}_{k \in \mathbb{Z}} \subset \mathcal{B}_{\varepsilon_{3} / 2}(O)$ of $L$, we can find $\mathbf{z} \in \mathcal{B}_{\varepsilon}\left(\mathbf{x}_{0}\right)$ such that $\left\{\mathbf{x}_{k}\right\}_{k \in \mathbb{Z}}$ is contained in the $\varepsilon$-neighborhood of

$$
\varphi\left(\mathbf{O}_{g}\left(\varphi^{-1}(\mathbf{z})\right) \cap \varphi^{-1}\left(\mathcal{B}_{\varepsilon_{3}}(O)\right)\right) .
$$

Take $\mathbf{v}_{0} \in \mathcal{B}_{\varepsilon_{3}}(O)$ with $\left|\mathbf{v}_{0}\right|=\varepsilon_{3} / 4\left(\varphi^{-1}\left(\mathbf{v}_{0}\right)\right.$ is the periodic point " $q$ " appearing in the first paragraph of this case). Since $l$ is the minimum period of $\mathbf{v}_{0}$ (see (5)), there is $0<\varepsilon_{4}<\varepsilon_{3} / 4$ such that

$$
\left(L^{i}\left(\mathcal{B}_{\varepsilon_{4}}\left(\mathbf{v}_{0}\right)\right) \cup L^{-i}\left(\mathcal{B}_{\varepsilon_{4}}\left(\mathbf{v}_{0}\right)\right)\right) \cap \mathcal{B}_{\varepsilon_{4}}\left(\mathbf{v}_{0}\right)=\emptyset \quad \text { for } 1 \leq i \leq l-1 .
$$

Let $\mathcal{J}=\left\{t \mathbf{v}_{0}: t \in\left[1,1+\varepsilon_{4} / 2\right]\right\} \subset \mathcal{B}_{\varepsilon_{4} / 2}\left(\mathbf{v}_{0}\right)$. Put $\varepsilon=\varepsilon_{4} / 5$, and let $0<\delta=\delta(\varepsilon)<\varepsilon$ be given by Claim 2.2. Then we can find a finite sequence $\left\{\mathbf{v}_{k}\right\}_{k=0}^{K} \subset \mathcal{J}$ (for some $K>0$ ) with $\mathbf{v}_{K}=\left(1+\varepsilon_{4} / 2\right) \mathbf{v}_{0}$ and $\left|\mathbf{v}_{k}-\mathbf{v}_{k+1}\right|<\delta$ for $0 \leq k \leq K-1$. Here the $\mathbf{v}_{k}$ are chosen so that if $\mathbf{v}_{k}=t_{k} \mathbf{v}_{0}$, then $t_{k}<t_{k+1}$ for $0 \leq k \leq K-1$. Define

$$
\begin{cases}\mathbf{x}_{k}=L^{k}\left(\mathbf{v}_{0}\right) & \text { for } k \leq 0, \\ \mathbf{x}_{k}=\mathbf{x}_{i l+j}=L^{j}\left(\mathbf{v}_{i}\right) & \text { for } 0 \leq i \leq K-1 \text { and } 0 \leq j \leq l-1, \\ \mathbf{x}_{k}=L^{k-l K}\left(\mathbf{v}_{K}\right) & \text { for } k \geq l K .\end{cases}
$$

Then $\left\{\mathbf{x}_{k}\right\}_{k \in \mathbb{Z}} \subset \bigcup_{i=0}^{l-1} L^{i}(\mathcal{J})$ is a $\delta$-pseudo-orbit of $L$ in $\mathcal{B}_{\varepsilon_{3} / 2}(O)$ by $(5)$. Hence there exists $\mathbf{z} \in \mathcal{B}_{\varepsilon}\left(\mathbf{x}_{0}\right)$ such that the pseudo-orbit is contained in the $\varepsilon$-neighborhood of $\varphi\left(\mathbf{O}_{g}\left(\varphi^{-1}(\mathbf{z})\right) \cap \varphi^{-1}\left(\mathcal{B}_{\varepsilon_{3}}(O)\right)\right)$ by Claim 2.2. It is easy to see that

$$
\varphi\left(\mathbf{O}_{g}\left(\varphi^{-1}(\mathbf{z})\right) \cap \varphi^{-1}\left(\mathcal{B}_{\varepsilon_{3}}(O)\right)\right)=\left\{L^{k}(\mathbf{z})\right\}_{k \in \mathbb{Z}}
$$

by (4) and (5). However, it follows from (5) and (6) that $L^{k}(\mathbf{z}) \cap \mathcal{B}_{\varepsilon}\left(\mathbf{x}_{l K}\right)=\emptyset$ for all $k \in \mathbb{Z}$. This is a contradiction and thus $p$ is hyperbolic.

As stated before, the case $n>1$ is similar. For completeness, we give a rough outline of the proof. Suppose that $f^{n}(p)=p(n>1)$ is not hyperbolic. By Lemma 1.1, we can prove an analog of Claim 1.1 (resp. Claim 2.1) for the periodic orbit $\mathbf{O}_{f}(p)=\left\{p, f(p), \ldots, f^{n-1}(p)\right\}$. Let $\varepsilon_{1}, g \in \mathcal{U}(f)$ and $L$ etc. be given by the analog. We may assume

$$
\left(g^{i}\left(B_{\varepsilon_{1}}(p)\right) \cup g^{-i}\left(B_{\varepsilon_{1}}(p)\right)\right) \cap B_{\varepsilon_{1}}(p)=\emptyset \quad \text { for } 1 \leq i \leq n-1
$$

by reducing $\varepsilon_{1}$ if necessary. Since $g$ has the weak shadowing property, the same property as stated in Claim 1.2 (resp. Claim 2.2) also holds for $L$ on a 
small neighborhood $\mathcal{B}$ of $\varphi\left(\mathbf{O}_{g}(p)\right)$. Fix $0<\varepsilon<\varepsilon_{1}$, and let $0<\delta=\delta(\varepsilon)<\varepsilon$ be given by the above property. Let $\left\{\mathbf{x}_{k}\right\}_{k \in \mathbb{Z}}$ be a $\delta$-pseudo-orbit of $L$ constructed in $\mathcal{B}$ (mimic the procedure used in the simple case). Then there exists $\mathbf{z} \in \mathcal{B}_{\varepsilon}\left(\mathbf{x}_{0}\right)$ such that the pseudo-orbit is contained in the $\varepsilon$-neighborhood of $\varphi\left(\mathbf{O}_{g}\left(\varphi^{-1}(\mathbf{z})\right) \cap \varphi^{-1}(\mathcal{B})\right)$. But it will be checked that the pseudo-orbit cannot be contained in the $\varepsilon$-neighborhood. This is a contradiction. The proof of $\operatorname{int} \mathcal{W} \mathcal{S}(M) \subset \mathcal{F}(M)$ is complete.

2. Proof of Proposition B. Suppose that $f \in \mathcal{A N}(M)$ and $\Omega(f)$ is finite. There is a subset $\left\{p_{1}, \ldots, p_{N}\right\} \subset \Omega(f)$ such that $\Omega(f)=\bigcup_{i=1}^{N} \mathbf{O}_{f}\left(p_{i}\right)$ and $\mathbf{O}_{f}\left(p_{i}\right) \cap \mathbf{O}_{f}\left(p_{j}\right)=\emptyset$ if $1 \leq i \neq j \leq N$. Clearly, $\mathbf{O}_{f}\left(p_{i}\right)$ is a periodic orbit. Since $\Omega(f)$ is hyperbolic, for all $p \in \Omega(f)$ and $\varepsilon>0$, there exist the local stable manifold $W_{\varepsilon}^{\mathrm{s}}(p)$ and the local unstable manifold $W_{\varepsilon}^{\mathrm{u}}(p)$. The stable manifold $W^{\mathrm{s}}(p)$ and the unstable manifold $W^{\mathrm{u}}(p)$ of $p$ are defined as usual. It is well known that

$$
M=\bigcup_{i=1}^{N} W^{\sigma}\left(\mathbf{O}_{f}\left(p_{i}\right)\right) \quad \text { for } \sigma=\mathrm{s}, \mathrm{u}
$$

and $W^{\mathrm{s}}\left(\mathbf{O}_{f}\left(p_{i}\right)\right) \cap W^{\mathrm{u}}\left(\mathbf{O}_{f}\left(p_{i}\right)\right)=\mathbf{O}_{f}\left(p_{i}\right)$ for $1 \leq i \leq N$. Here $W^{\sigma}\left(\mathbf{O}_{f}\left(p_{i}\right)\right)=$ $\bigcup\left\{W^{\sigma}(p): p \in \mathbf{O}_{f}\left(p_{i}\right)\right\}(\sigma=\mathrm{s}, \mathrm{u})$.

Under the no-cycle condition, one can choose a simple ordering " $<$ " on the $p_{i}$ such that $p_{1}<\ldots<p_{N}$, and $p_{i}<p_{j}$ implies that

$$
W^{\mathrm{s}}\left(\mathbf{O}_{f}\left(p_{j}\right)\right) \cap W^{\mathrm{u}}\left(\mathbf{O}_{f}\left(p_{i}\right)\right)=\emptyset .
$$

Thus there exists a sequence of compact sets $\emptyset=M_{0} \subset M_{1} \subset \ldots \subset M_{N}=$ $M$ (which is called a filtration for $f$; cf. [13]) such that for $1 \leq i \leq N$,

$$
f\left(M_{i}\right) \subset \operatorname{int} M_{i} \text { and } \bigcap_{m \in \mathbb{Z}} f^{m}\left(M_{i} \backslash M_{i-1}\right)=\mathbf{O}_{f}\left(p_{i}\right) .
$$

Suppose further that $f \in \operatorname{int} \mathcal{W S}(M)$. Our aim is to show that $f$ satisfies the strong transversality condition; that is, for every $x \in M$, there are $1 \leq i \neq j \leq N$ such that $T_{x} M=T_{x} W^{\mathrm{s}}\left(\mathbf{O}_{f}\left(p_{i}\right)\right)+T_{x} W^{\mathrm{u}}\left(\mathbf{O}_{f}\left(p_{j}\right)\right)$ $\left(x \in W^{\mathrm{s}}\left(\mathbf{O}_{f}\left(p_{i}\right)\right) \cap W^{\mathrm{u}}\left(\mathbf{O}_{f}\left(p_{j}\right)\right)\right)$. Assuming that there is $x \in W^{\mathrm{s}}\left(\mathbf{O}_{f}\left(p_{i}\right)\right) \cap$ $W^{\mathrm{u}}\left(\mathbf{O}_{f}\left(p_{j}\right)\right)(1 \leq i<j \leq N)$ with

$$
T_{x} M \neq T_{x} W^{\mathrm{s}}\left(\mathbf{O}_{f}\left(p_{i}\right)\right)+T_{x} W^{\mathrm{u}}\left(\mathbf{O}_{f}\left(p_{j}\right)\right),
$$

we shall get a contradiction. Without loss of generality, we may suppose $x \in W^{\mathrm{s}}\left(p_{i}\right) \cap W^{\mathrm{u}}\left(p_{j}\right)$. Clearly, both $p_{i}$ and $p_{j}\left(p_{i} \neq p_{j}\right)$ are saddles.

Let $n_{i}$ and $n_{j}$ be their (minimum) periods; that is, $f^{n_{i}}\left(p_{i}\right)=p_{i}, f^{n_{j}}\left(p_{j}\right)$ $=p_{j}$. For the sake of simplicity, assume $n_{i}=n_{j}=1$ (although the other case is treated similarly, a rough outline of the proof will be given later on). 
SteP 1. In this step, linearizing $f$ at suitable points with a small perturbation, we make a new diffeomorphism $g \in \mathcal{W S}(M)\left(C^{1}\right.$-near $\left.f\right)$. Then we induce a locally defined linear system (from $g$ ) with the same geometric structure as Plamenevskaya's map. First, applying Lemma 1.1 to

$$
F_{q}=\varphi_{q} \circ f \circ \varphi_{q}^{-1}: \varphi_{q}\left(U_{\beta}(q)\right) \rightarrow \mathbb{R}^{2} \quad\left(q \in\left\{p_{i}, p_{j}\right\}\right)
$$

we linearize $f$ at $p_{i}$ and $p_{j}$ simultaneously. To simplify notation, suppose that $\varphi_{p_{i}} \neq \varphi_{p_{j}}$ and $\varphi_{q}(q)=O_{q} \in \mathbb{R}^{2}\left(q \in\left\{p_{i}, p_{j}\right\}\right)$, where $O_{q}$ are the origins (the other case is treated similarly).

Lemma 2.1. For any $C^{1}$ neighborhood $\mathcal{U}(f) \subset \mathcal{W S}(M)$ and $q \in\left\{p_{i}, p_{j}\right\}$, let $\varepsilon_{0}, \delta_{0}>0$ be given by Lemma $1.1\left(B_{4 \varepsilon_{0}}\left(p_{i}\right) \cap B_{4 \varepsilon_{0}}\left(p_{j}\right)=\emptyset\right)$. Then there are linear isomorphisms $\mathcal{O}_{q}: \mathbb{R}^{2} \rightarrow \mathbb{R}^{2}$ with $\left\|\mathcal{O}_{q}-I\right\|<\delta_{0}\left(q \in\left\{p_{i}, p_{j}\right\}\right)$ and $g \in \mathcal{U}(f)$ satisfying

(i) $g(y)=f(y)$ if $y \in\left\{p_{i}, p_{j}\right\} \cup M \backslash\left(B_{4 \varepsilon_{0}}\left(p_{i}\right) \cup B_{4 \varepsilon_{0}}\left(p_{j}\right)\right)$,

(ii) there exists $0<\varepsilon_{1}<\varepsilon_{0} / 4$ such that for $q \in\left\{p_{i}, p_{j}\right\}, B_{2 \varepsilon_{1}}(q) \subset$ $g\left(B_{\varepsilon_{0}}(q)\right) \cap g^{-1}\left(B_{\varepsilon_{0}}(q)\right)$ and $g_{\mid B_{2 \varepsilon_{1}}(q)}$ (resp. $\left.g_{\mid B_{2 \varepsilon_{1}}(q)}^{-1}\right)$ is conjugate to

$$
L_{q}=\mathcal{O}_{q} \circ D_{O_{q}} F_{q_{\mid \varphi\left(B_{2 \varepsilon_{1}}(q)\right)}} \quad\left(\text { resp. } L_{q}^{-1}=\left(\mathcal{O}_{q} \circ D_{O_{q}} F_{q}\right)_{\mid \varphi\left(B_{2 \varepsilon_{1}}(q)\right)}^{-1}\right),
$$

(iii) if we extend $L_{q}\left(q \in\left\{p_{i}, p_{j}\right\}\right)$ to linear isomorphisms on $\mathbb{R}^{2}$, and if we denote the eigenvalues of $L_{p_{j}}$ by $\lambda, \kappa(0<|\lambda|<1,|\kappa|>1)$ and the eigenvalues of $L_{p_{i}}$ by $\mu, \nu(|\mu|>1,0<|\nu|<1)$, then both $\log |\lambda|$ and $\log |\mu|$ are rational.

In the above lemma, since $\varepsilon_{0}$ is small enough, we may assume

$$
\left(B_{\varepsilon_{0}}(x) \cup \Omega(f)\right) \cap\left(B_{4 \varepsilon_{0}}\left(p_{i}\right) \cup B_{4 \varepsilon_{0}}\left(p_{j}\right)\right)=\left\{p_{i}, p_{j}\right\} .
$$

Thus $\Omega(g)=\Omega(f)$ since $f$ is $\Omega$-stable.

For $q \in\left\{p_{i}, p_{j}\right\}$, let $E_{q}^{\mathrm{s}} \oplus E_{q}^{\mathrm{u}}$ be the hyperbolic splitting for $L_{q}$ (where $E_{q}^{\mathrm{s}}$ and $E_{q}^{\mathrm{u}}$ are the stable and unstable eigenspaces respectively), and set

$$
E_{q, \varepsilon}^{\sigma}=\left\{\mathbf{w} \in E_{q}^{\sigma}:|\mathbf{w}| \leq \varepsilon\right\} \quad \text { and } \quad E_{q, \varepsilon}^{\sigma}(\mathbf{v})=E_{q, \varepsilon}^{\sigma}+\mathbf{v} \quad(\sigma=\mathrm{s}, \mathrm{u})
$$

for $\varepsilon>0, \mathbf{v} \in \mathbb{R}^{2}$. For convenience, put

$$
W_{\varepsilon}^{\sigma}(q, g)=\varphi^{-1}\left(E_{q, \varepsilon}^{\sigma}\right) \quad\left(\sigma=\mathrm{s}, \mathrm{u} \text { and } 0<\varepsilon \leq 2 \varepsilon_{1}\right) .
$$

For $q \in\left\{p_{i}, p_{j}\right\}$, denote by $W^{\sigma}(q, g)(\sigma=\mathrm{s}, \mathrm{u})$ the stable and unstable manifolds for $g$. In general, $x \notin W^{\mathrm{s}}\left(p_{i}, g\right) \cap W^{\mathrm{u}}\left(p_{j}, g\right)$. However, since $\mathcal{U}(f)$ is arbitrary, perturbing $g$ (at $x$ ) if necessary, we may suppose that $x \in$ $W^{\mathrm{s}}\left(p_{i}, g\right) \cap W^{\mathrm{u}}\left(p_{j}, g\right)$ and

$$
T_{x} M \neq T_{x} W^{\mathrm{s}}\left(p_{i}, g\right)+T_{x} W^{\mathrm{u}}\left(p_{j}, g\right)
$$

(see [10, Lemma 3]).

Fix $l>0$ such that $x \in g^{-l}\left(W_{\varepsilon_{1} / 2}^{\mathrm{s}}\left(p_{i}, g\right)\right) \cap g^{l}\left(W_{\varepsilon_{1} / 2}^{\mathrm{u}}\left(p_{j}, g\right)\right)$. To simplify notation, we consider the case $l=1$ (the other case is treated similarly). 
Thus, the non-transversality at $x$ implies

$$
D_{g^{-1}(x)} g^{2}\left(T_{g^{-1}(x)} W_{\varepsilon_{1} / 2}^{\mathrm{u}}\left(p_{j}, g\right)\right)=T_{g(x)} W_{\varepsilon_{1} / 2}^{\mathrm{s}}\left(p_{i}, g\right) .
$$

Pick $0<\varepsilon_{2}<\varepsilon_{1}$ such that

$$
B_{\varepsilon_{2}}\left(g^{-1}(x)\right) \subset B_{\varepsilon_{1}}\left(p_{j}\right) \cap g^{-1}\left(U_{\beta}(x)\right) \cap g^{-2}\left(B_{\varepsilon_{1}}\left(p_{i}\right)\right) .
$$

Let us perturb $g$ at $g^{-1}(x)$ and $x$ mimicking the proof of [2, Lemma 1.1]. Put

$$
\begin{aligned}
G_{g^{-1}(x)} & =\varphi_{x} \circ g \circ \varphi_{p_{j}}^{-1}: \varphi_{p_{j}}\left(U_{\varepsilon_{2}}\left(g^{-1}(x)\right)\right) \rightarrow \mathbb{R}^{2}, \\
G_{x} & =\varphi_{p_{i}} \circ g \circ \varphi_{x}^{-1}: \varphi_{x}\left(g\left(U_{\varepsilon_{2}}\left(g^{-1}(x)\right)\right)\right) \rightarrow \mathbb{R}^{2} .
\end{aligned}
$$

Lemma 2.2. Fix a $C^{1}$ neighborhood $\mathcal{U}(g) \subset \mathcal{U}(f)$, and let $\varepsilon_{2}>0$ be as above. Then there are $0<\varepsilon_{3}<\varepsilon_{2} / 4$ and $g_{1} \in \mathcal{U}(g)$ such that

(i) $B_{4 \varepsilon_{3}}\left(g^{-1}(x)\right) \cap B_{4 \varepsilon_{3}}(x)=\emptyset$ and $\Omega(g) \cap\left(B_{4 \varepsilon_{3}}\left(g^{-1}(x)\right) \cup B_{4 \varepsilon_{3}}(x)\right)=\emptyset$,

(ii) $g_{1}(y)=g(y)$ if $y \in\left\{g^{-1}(x), x\right\} \cup\left\{M \backslash\left(B_{4 \varepsilon_{3}}\left(g^{-1}(x)\right) \cup B_{4 \varepsilon_{3}}(x)\right)\right\}$,

(iii) $\varphi \circ g_{1}=L_{g^{-1}(x)} \circ \varphi_{p_{j}}$ on $B_{\varepsilon_{3}}\left(g^{-1}(x)\right)$ and $\varphi_{p_{i}} \circ g_{1}=L_{x} \circ \varphi$ on $B_{\varepsilon_{3}}(x)$, where

$$
\begin{cases}L_{g^{-1}(x)}(\mathbf{v})=\varphi(x)+D_{\varphi_{p_{j}}\left(g^{-1}(x)\right)} G_{g^{-1}(x)}\left(\mathbf{v}-\varphi_{p_{j}}\left(g^{-1}(x)\right)\right) & \\ & \text { if } \mathbf{v} \in \varphi_{p_{j}}\left(B_{\varepsilon_{3}}\left(g^{-1}(x)\right)\right), \\ L_{x}(\mathbf{v})=\varphi_{p_{i}}(g(x))+D_{\varphi(x)} G_{x}(\mathbf{v}-\varphi(x)) & \text { if } \mathbf{v} \in \varphi_{x}\left(B_{\varepsilon_{3}}(x)\right)\end{cases}
$$

In the above lemma, since $\varepsilon_{3}$ is small enough, we may assume

$$
B_{4 \varepsilon_{3}}\left(g^{-1}(x)\right) \cap g_{1}^{-1}\left(B_{4 \varepsilon_{3}}\left(g^{-1}(x)\right)\right)=\emptyset .
$$

Hence, if we denote the set $W_{\varepsilon_{1}}^{\mathrm{u}}\left(p_{j}, g\right) \cap B_{\varepsilon_{3}}\left(g^{-1}(x)\right)$ by $C_{g^{-1}(x)}^{\mathrm{u}}$, then $g_{1}^{-n}\left(C_{g^{-1}(x)}^{\mathrm{u}}\right)=g^{-n}\left(C_{g^{-1}(x)}^{\mathrm{u}}\right)$ for all $n \geq 0$.

Take $0<\varepsilon_{4}<\varepsilon_{3}$ such that $g_{1}\left(B_{\varepsilon_{4}}\left(g_{1}^{-1}(x)\right)\right) \subset B_{\varepsilon_{3}}(x)$. Then $L_{x} \circ$ $L_{g^{-1}(x)}: \varphi_{p_{j}}\left(B_{\varepsilon_{4}}\left(g_{1}^{-1}(x)\right)\right) \rightarrow \mathbb{R}^{2}$ is well defined and

$$
\varphi_{p_{i}} \circ g_{1}^{2}=L_{x} \circ L_{g^{-1}(x)} \circ \varphi_{p_{j}} \quad \text { on } B_{\varepsilon_{4}}\left(g_{1}^{-1}(x)\right) .
$$

Note that by Lemma 2.2(i), $\Omega\left(g_{1}\right)=\Omega(g)$ since $g$ is also $\Omega$-stable.

Hereafter, we denote $g_{1}$ and $W^{\sigma}\left(x, g_{1}\right)$ by $g$ and $W^{\sigma}(x)(\sigma=\mathrm{s}, \mathrm{u})$ etc. for simplicity. Obviously, $\varphi(g(x)) \in E_{p_{i}}^{\mathrm{s}}$ and $\varphi\left(g^{-1}(x)\right) \in E_{p_{j}}^{\mathrm{u}}$. Fix $\varepsilon>0$ such that $E_{p_{j}, \varepsilon}^{\sigma}\left(\varphi\left(g^{-1}(x)\right)\right) \subset \varphi\left(B_{\varepsilon_{4}}\left(g^{-1}(x)\right)\right)(\sigma=s, u)$. Then, by (8) and (9), we conclude that

$$
\begin{aligned}
& L_{x} \circ L_{g^{-1}(x)}\left(E_{p_{j}, \varepsilon}^{\mathrm{u}}\left(\varphi\left(g^{-1}(x)\right)\right)\right) \subset E_{p_{i}}^{\mathrm{s}}, \\
& L_{x} \circ L_{g^{-1}(x)}\left(E_{p_{j}, \varepsilon}^{\mathrm{s}}\left(\varphi\left(g^{-1}(x)\right)\right)\right) \neq E_{p_{i}}^{\mathrm{s}}
\end{aligned}
$$

(see the figure). 


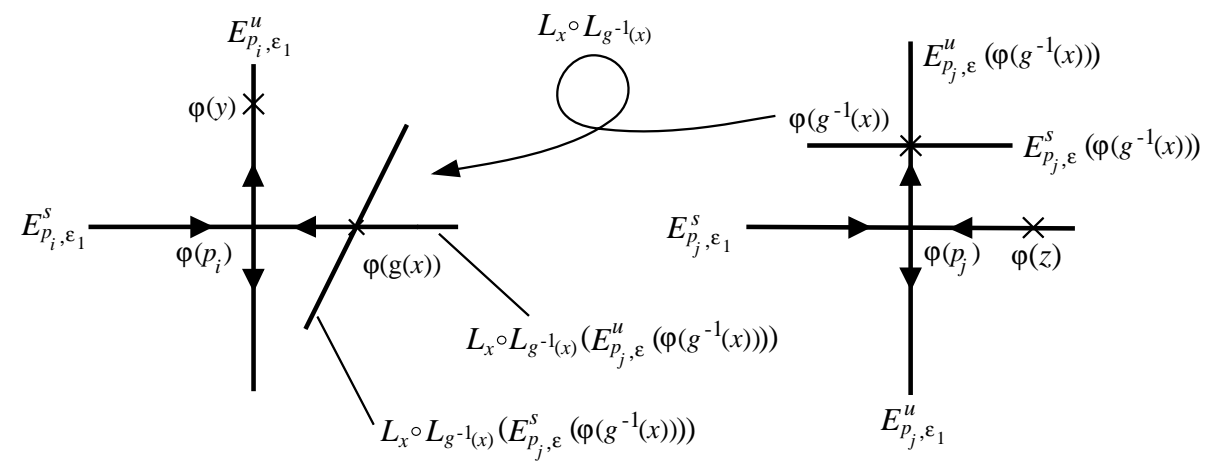

We sometimes denote a point $\mathbf{v} \in \mathbb{R}^{2}$ by coordinates $(v, w)$ with respect to $E_{q}^{\mathrm{s}} \times E_{q}^{\mathrm{u}}$ if $\mathbf{v} \in \varphi\left(B_{\varepsilon_{0}}(q)\right)\left(q \in\left\{p_{i}, p_{j}\right\}\right)$. Let

$$
\begin{gathered}
\varphi(g(x))=\left(v_{g(x)}, 0\right) \in E_{p_{i}}^{\mathrm{s}} \times E_{p_{i}}^{\mathrm{u}}, \\
\varphi\left(g^{-1}(x)\right)=\left(0, w_{g^{-1}(x)}\right) \in E_{p_{j}}^{\mathrm{s}} \times E_{p_{j}}^{\mathrm{u}} .
\end{gathered}
$$

Then there are constants $a, b$ and $c$ such that

$$
L_{x} \circ L_{g^{-1}(x)}\left(\begin{array}{c}
v \\
w
\end{array}\right)=\left(\begin{array}{cc}
a & b \\
c & 0
\end{array}\right)\left(\begin{array}{c}
v \\
w
\end{array}\right)+\left(\begin{array}{c}
v_{g(x)}-b w_{g^{-1}(x)} \\
0
\end{array}\right) \in E_{p_{j}}^{\mathrm{s}} \times E_{p_{j}}^{\mathrm{u}}
$$
if $(v, w) \in \varphi\left(B_{\varepsilon_{4}}\left(g^{-1}(x)\right)\right)$. Obviously, $c \neq 0$.

Finally, we define a system consisting of $L_{p_{i}}, L_{g^{-1}(x)}, L_{x}$ and $L_{p_{j}}$ by

$$
\mathcal{L}_{g}(\mathbf{v})= \begin{cases}L_{p_{j}}(\mathbf{v}) & \text { if } \mathbf{v} \in \varphi\left(B_{\varepsilon_{1}}\left(p_{j}\right) \backslash B_{\varepsilon_{4}}\left(g^{-1}(x)\right)\right) \\ L_{g^{-1}(x)}(\mathbf{v}) & \text { if } \mathbf{v} \in \varphi\left(B_{\varepsilon_{4}}\left(g^{-1}(x)\right)\right) \\ L_{x}(\mathbf{v}) & \text { if } \mathbf{v} \in \varphi\left(g\left(B_{\varepsilon_{4}}\left(g^{-1}(x)\right)\right)\right) \\ L_{p_{i}}(\mathbf{v}) & \text { if } \mathbf{v} \in \varphi\left(B_{\varepsilon_{1}}\left(p_{i}\right)\right)\end{cases}
$$

and

$$
\mathcal{L}_{g}^{-1}(\mathbf{v})= \begin{cases}L_{p_{j}}^{-1}(\mathbf{v}) & \text { if } \mathbf{v} \in \varphi\left(B_{\varepsilon_{1}}\left(p_{j}\right)\right) \\ L_{x}^{-1}(\mathbf{v}) & \text { if } \mathbf{v} \in \varphi\left(g^{2}\left(B_{\varepsilon_{4}}\left(g^{-1}(x)\right)\right)\right) \\ L_{g^{-1}(x)}^{-1}(\mathbf{v}) & \text { if } \mathbf{v} \in \varphi\left(g\left(B_{\varepsilon_{4}}\left(g^{-1}(x)\right)\right)\right) \\ L_{p_{i}}^{-1}(\mathbf{v}) & \text { if } \mathbf{v} \in \varphi\left(B_{\varepsilon_{1}}\left(p_{i}\right) \backslash g^{2}\left(B_{\varepsilon_{4}}\left(g^{-1}(x)\right)\right)\right) .\end{cases}
$$

Notice that if $\mathbf{v}$ belongs to the domain of $\mathcal{L}_{g}\left(\right.$ resp. $\left.\mathcal{L}_{g}^{-1}\right)$, then

$$
\varphi \circ g \circ \varphi^{-1}(\mathbf{v})=\mathcal{L}_{g}(\mathbf{v}) \quad\left(\operatorname{resp} . \varphi \circ g^{-1} \circ \varphi^{-1}(\mathbf{v})=\mathcal{L}_{g}^{-1}(\mathbf{v})\right) .
$$

REMARK. We can see that the system $\mathcal{L}_{g}$ closely resembles Plamenevskaya's map, and that the dynamics of $g$ on the neighborhood of $\left\{p_{i}, p_{j}\right.$, $\left.g^{-1}(x), x\right\}$ coincides with the dynamics of $\mathcal{L}_{g}$ on the neighborhood of $\left\{\varphi\left(p_{i}\right), \varphi\left(p_{j}\right), \varphi\left(g^{-1}(x)\right), \varphi(x)\right\}$. Unfortunately, in the proof of Proposition B we cannot restrict ourselves to $\mathcal{L}_{g}$, because we must control the global behavior of a weakly shadowing orbit for $g$. But we consider $\mathcal{L}_{g}$ (instead of $g$ ) when the orbit visits the above neighborhood. 
STEP 2. Let $g$ be the map constructed in Step 1. In this step, we prepare two lemmas to control the behavior of a weakly shadowing orbit for $g$. For any $\mathbf{v} \in E_{q}^{\mathrm{s}} \times E_{q}^{\mathrm{u}}$ and $\varepsilon>0$, put

$$
\mathcal{B}_{\varepsilon}(\mathbf{v})=E_{q, \varepsilon}^{\mathrm{s}}(\mathbf{v}) \times E_{q, \varepsilon}^{\mathrm{u}}(\mathbf{v}) \quad\left(q \in\left\{p_{i}, p_{j}\right\}\right),
$$

and furthermore, define

$$
B_{\varepsilon}^{\prime}(v)=\varphi^{-1}\left(\mathcal{B}_{\varepsilon}(\varphi(v))\right)
$$

if $\varphi(v)(v \in M)$ is contained in the interior of the domain of $\mathcal{L}_{g}$ and if $\varepsilon$ is small enough. Clearly, $B_{\varepsilon}^{\prime}(v)$ is a closed neighborhood of $v$ in $M$. We may assume that $B_{2 \varepsilon_{1}}^{\prime}(q) \subset B_{\varepsilon_{0}}(q)\left(q \in\left\{p_{i}, p_{j}\right\}\right)$ by reducing $\varepsilon_{1}$ if necessary (see Lemma 2.1(ii)).

Recall that $\Omega(g)=\Omega(f)$ by construction. It is not hard to show that the sequence $\emptyset=M_{0} \subset M_{1} \subset \ldots \subset M_{N}=M$ is a filtration for $g$ (since $g$ is $C^{1}$-near $f$ ). Thus $g\left(M_{k}\right) \subset \operatorname{int} M_{k}$ for $1 \leq k \leq N$, and for any neighborhood $U$ of $\mathbf{O}_{g}\left(p_{k}\right)(1 \leq k \leq N)$, there exists a positive integer $m_{k}$ satisfying $\mathbf{O}_{g}\left(p_{k}\right) \subset g^{m_{k}}\left(M_{k}\right) \backslash g^{-m_{k}}\left(M_{k-1}\right) \subset U$. Since $\varepsilon_{1}$ is small enough, we may suppose

$$
B_{2 \varepsilon_{1}}^{\prime}\left(p_{k}\right) \subset \operatorname{int} M_{k} \backslash M_{k-1} \quad \text { for } k=i, j .
$$

For $q \in\left\{p_{i}, p_{j}\right\}$, put

$$
D^{\mathrm{s}}(q)=\overline{W_{\varepsilon_{1}}^{\mathrm{s}}(q) \backslash g\left(W_{\varepsilon_{1}}^{\mathrm{s}}(q)\right)} \quad \text { and } \quad D^{\mathrm{u}}(q)=\overline{W_{\varepsilon_{1}}^{\mathrm{u}}(q) \backslash g^{-1}\left(W_{\varepsilon_{1}}^{\mathrm{u}}(q)\right)} .
$$

Then $D^{\mathrm{s}}(q)$ (resp. $D^{\mathrm{u}}(q)$ ) is a fundamental domain for the stable (resp. unstable) manifold of $q$. Clearly, $q \notin D^{\mathrm{s}}(q) \cup D^{\mathrm{u}}(q)$ for $q \in\left\{p_{i}, p_{j}\right\}$. Thus, by the filtration, there exists $m>0$ such that for $k=i, j$,

$$
g^{m}\left(D^{\mathrm{u}}\left(p_{k}\right)\right) \subset \operatorname{int} M_{k-1} \quad \text { and } \quad g^{-m}\left(D^{\mathrm{s}}\left(p_{k}\right)\right) \subset \operatorname{int} M_{k+1} \backslash M_{k} .
$$

Lemma 2.3. There exists $0<\varepsilon_{5}<\varepsilon_{4}$ such that for all $k \geq 2$,

(i) $d\left(g^{k}\left(B_{\varepsilon_{5}}^{\prime}\left(D^{\mathrm{u}}\left(p_{i}\right)\right)\right), B_{\varepsilon_{5}}^{\prime}\left(W_{\varepsilon_{1}}^{\mathrm{u}}\left(p_{i}\right)\right)\right)>\varepsilon_{5}$,

(ii) $d\left(g^{-k}\left(B_{\varepsilon_{5}}^{\prime}\left(D^{\mathrm{s}}\left(p_{j}\right)\right)\right), B_{\varepsilon_{5}}^{\prime}\left(W_{\varepsilon_{1}}^{\mathrm{s}}\left(p_{j}\right)\right)\right)>\varepsilon_{5}$.

Proof. Let $m>0$ be as in (15). We prove assertion (i) ((ii) follows similarly). Suppose that for every $n>0$, there are $x_{n} \in B_{1 / n}^{\prime}\left(D^{\mathrm{u}}\left(p_{i}\right)\right)$ and $k_{n} \geq 2$ such that $g^{k_{n}}\left(x_{n}\right) \in B_{2 / n}^{\prime}\left(W_{\varepsilon_{1}}^{\mathrm{u}}\left(p_{i}\right)\right)$. If $k_{n} \leq m$ for all $n$, then there exists $2 \leq m^{\prime} \leq m$ such that $g^{m^{\prime}}(x) \in W_{\varepsilon_{1}}^{\mathrm{u}}\left(p_{i}\right)$. Here $x=\lim _{n \rightarrow \infty} x_{n} \in$ $D^{\mathrm{u}}\left(p_{i}\right)$. Thus $x \in g^{-m^{\prime}}\left(W_{\varepsilon_{1}}^{\mathrm{u}}\left(p_{i}\right)\right) \cap D^{\mathrm{u}}\left(p_{i}\right) \neq \emptyset$. This is a contradiction since $m^{\prime} \geq 2$. Thus $k_{n} \rightarrow \infty$ as $n \rightarrow \infty$. By (15), if we take $n$ large enough, then $g^{m}\left(x_{n}\right) \in M_{i-1}$ (since $x_{n} \rightarrow x \in D^{\mathrm{u}}\left(p_{i}\right)$ as $n \rightarrow \infty$ ). On the other hand, if $n$ is so large that $k_{n}>m$, then $g^{k_{n}}\left(x_{n}\right)=g^{k_{n}-m}\left(g^{m}\left(x_{n}\right)\right) \in M_{i-1}$ since $g\left(M_{i-1}\right) \subset M_{i-1}$. This is also a contradiction, because $\lim _{n \rightarrow \infty} g^{k_{n}}\left(x_{n}\right) \in$ $W_{\varepsilon_{1}}^{\mathrm{u}}\left(p_{i}\right) \subset M_{i} \backslash M_{i-1}$ by (14). 
The next lemma is almost clear since the map $g$ is conjugate to the (locally defined) linear isomorphism $L_{q}$ on the neighborhood of $q$.

Lemma 2.4 (cf. [4]). For $q \in\left\{p_{i}, p_{j}\right\}$ and $0<\varepsilon<\varepsilon_{1}$, put

$$
\begin{aligned}
& V_{\varepsilon}^{\mathrm{s}}(q)=W_{2 \varepsilon_{1}}^{\mathrm{u}}(q) \cup\left(\bigcup_{k=0}^{\infty} g^{k}\left(B_{\varepsilon}^{\prime}\left(D^{\mathrm{s}}(q)\right)\right) \cap B_{2 \varepsilon_{1}}^{\prime}(q)\right), \\
& V_{\varepsilon}^{\mathrm{u}}(q)=W_{2 \varepsilon_{1}}^{\mathrm{s}}(q) \cup\left(\bigcup_{k=0}^{\infty} g^{-k}\left(B_{\varepsilon}^{\prime}\left(D^{\mathrm{u}}(q)\right)\right) \cap B_{2 \varepsilon_{1}}^{\prime}(q)\right) .
\end{aligned}
$$

Then $V_{\varepsilon}^{\sigma}(q)$ is a neighborhood of $W_{\varepsilon_{1}}^{\mathrm{s}}(q) \cup W_{\varepsilon_{1}}^{\mathrm{u}}(q)$ for $\sigma=\mathrm{s}, \mathrm{u}$.

Proof. For $q \in\left\{p_{i}, p_{j}\right\}$ and $0<\varepsilon<\varepsilon_{1}$, by formulae (7), (12) and (13),

$$
\left.\varphi\left(V_{\varepsilon}^{\mathrm{s}}(q)\right)=E_{q, 2 \varepsilon_{1}}^{\mathrm{u}} \cup\left(\bigcup_{k=0}^{\infty} \mathcal{L}_{g}^{k}\left(\mathcal{B}_{\varepsilon}\left(\varphi\left(D^{\mathrm{s}}(q)\right)\right)\right) \cap \mathcal{B}_{2 \varepsilon_{1}}\left(O_{q}\right)\right)\right),
$$

and clearly, this is a neighborhood of $\varphi\left(W_{\varepsilon_{1}}^{\mathrm{s}}(q) \cup W_{\varepsilon_{1}}^{\mathrm{u}}(q)\right)=E_{q, \varepsilon_{1}}^{\mathrm{s}} \cup E_{q, \varepsilon_{1}}^{\mathrm{u}}$. The assertion on $V_{\varepsilon}^{\mathrm{u}}(q)$ follows similarly.

Proof of Proposition B. Let $g \in \mathcal{W S}(M)$ and $\mathcal{L}_{g}$ be as in Step 1. Under the above preliminaries, by Plamenevskaya's technique we shall obtain a contradiction.

Recall that $x \in g^{-1}\left(W_{\varepsilon_{1} / 2}^{\mathrm{s}}\left(p_{i}\right)\right) \cap g\left(W_{\varepsilon_{1} / 2}^{\mathrm{u}}\left(p_{j}\right)\right)$, and let $\varepsilon_{5}>0$ be given by Lemma 2.3. Take $0<\varepsilon_{6}<\varepsilon_{5}$ such that $B_{\varepsilon_{6}}^{\prime}(g(x)) \subset V_{\varepsilon_{5}}^{\mathrm{u}}\left(p_{i}\right)$ and $B_{\varepsilon_{6}}^{\prime}(x) \subset$ $B_{\varepsilon_{0}}(x)$, and fix $0<\varepsilon_{7}<\varepsilon_{6}$ satisfying

$$
B_{\varepsilon_{7}}^{\prime}\left(g^{-1}(x)\right) \subset g^{-1}\left(B_{\varepsilon_{6}}^{\prime}(x)\right) \cap g^{-2}\left(B_{\varepsilon_{6}}^{\prime}(g(x))\right) \cap V_{\varepsilon_{5}}^{\mathrm{s}}\left(p_{j}\right) .
$$

Let $\lambda$ and $\mu$ be as in Lemma 2.1(iii). For simplicity, we deal with the case when both $\lambda$ and $\mu$ are positive (the other case is treated similarly). Since both $\log \lambda$ and $\log \mu$ are rational,

$$
\frac{\log \lambda}{\log \mu}=-\frac{r}{s}
$$

for some integers $r, s>0$. Put $0<\gamma=e^{-(\log \mu) / s}<1$. Then $\lambda=\gamma^{r}$ and $\mu=\gamma^{-s}$.

Let $c$ be as in (11), and assume $c>0$ (the other case is treated similarly). The proof is divided into two cases.

CASE 1: $c>1$. Take $\left(0, w_{y}\right) \in E_{p_{i}, \varepsilon_{1}}^{\mathrm{u}}$ and $\left(v_{z}, 0\right) \in E_{p_{j}, \varepsilon_{1}}^{\mathrm{s}}$ such that $w_{y}=\varepsilon_{1} \gamma^{1 / 2}$ and $v_{z}=\varepsilon_{1} c^{-1}$. Set

$$
y=\varphi^{-1}\left(0, w_{y}\right) \in W_{\varepsilon_{1}}^{\mathrm{u}}\left(p_{i}\right) \quad \text { and } \quad z=\varphi^{-1}\left(v_{z}, 0\right) \in W_{\varepsilon_{1}}^{\mathrm{s}}\left(p_{j}\right),
$$

and fix $0<\varepsilon<\min \left\{\varepsilon_{7}, \varepsilon_{1}(2 c)^{-1} \gamma^{1 / 2}\left(1-\gamma^{1 / 2}\right)\right\}$ such that

$$
B_{\varepsilon}^{\prime}(y) \cap W_{2 \varepsilon_{1}}^{\mathrm{s}}\left(p_{i}\right)=\emptyset \quad \text { and } \quad B_{\varepsilon}^{\prime}(z) \cap W_{2 \varepsilon_{1}}^{\mathrm{u}}\left(p_{j}\right)=\emptyset \text {. }
$$


Pick $0<\varepsilon^{\prime}<\varepsilon$ so small that $B_{\varepsilon^{\prime}}(v) \subset B_{\varepsilon}^{\prime}(v)$ for all $v \in\left\{g^{-1}(x), x, y, z\right\}$. Let $0<\delta=\delta\left(\varepsilon^{\prime}\right)<\varepsilon^{\prime}$ be as in the weak shadowing property of $g$, and choose an integer $k>0$ such that

$$
\max \left\{d\left(g^{-k}(y), g^{k}(x)\right), d\left(g^{-k}(x), g^{k}(z)\right)\right\}<\delta .
$$

Denote by $\mathcal{P} \mathcal{O}_{\delta}$ the following $\delta$-pseudo-orbit of $g$ consisting of three pieces of $g$-orbits:

$$
\begin{aligned}
& \left\{\ldots, g^{-2}(z), g^{-1}(z), z, g(z), \ldots, g^{k-1}(z), g^{-k}(x), g^{-k-1}(x), \ldots, g^{-1}(x),\right. \\
& \left.\quad x, g(x), \ldots, g^{k-1}(x), g^{-k}(y), g^{-k+1}(y), \ldots, g^{-1}(y), y, g(y), g^{2}(y), \ldots\right\} .
\end{aligned}
$$

Since $g$ has the weak shadowing property, there exists $w \in B_{\varepsilon}^{\prime}\left(g^{-1}(x)\right)$ weakly $\varepsilon^{\prime}$-shadowing $\mathcal{P} \mathcal{O}_{\delta}$ (recall (13) and the choice of $\varepsilon^{\prime}$ ). It is easy to see that

$$
g(w) \notin B_{\varepsilon}^{\prime}(z) \cup B_{\varepsilon}^{\prime}(y), \quad \mathbf{O}_{g}^{+}\left(g^{2}(w)\right) \cap B_{\varepsilon}^{\prime}(z)=\emptyset, \quad \mathbf{O}_{g}^{-}(w) \cap B_{\varepsilon}^{\prime}(y)=\emptyset,
$$

where

$$
\mathbf{O}_{g}^{+}\left(g^{2}(w)\right)=\left\{g^{k}\left(g^{2}(w)\right)\right\}_{k \geq 0} \quad \text { and } \quad \mathbf{O}_{g}^{-}(w)=\left\{g^{-k}(w)\right\}_{k \geq 0} .
$$

For, since $g(w) \in B_{\varepsilon_{6}}^{\prime}(x)$, we have $g(w) \notin B_{\varepsilon}^{\prime}(z) \cup B_{\varepsilon}^{\prime}(y)$ (see the paragraph following Lemma 2.1). Since $g^{m}\left(g^{2}(w)\right) \in M_{i}$ for all $m \geq 0$ and $B_{\varepsilon}^{\prime}(z) \subset B_{2 \varepsilon_{1}}^{\prime}\left(p_{j}\right) \subset M_{j} \backslash M_{i}$ (see (14) and (15)), we have $\mathbf{O}_{g}^{+}\left(g^{2}(w)\right) \cap$ $B_{\varepsilon}^{\prime}(z)=\emptyset$. Similarly, $\mathbf{O}_{g}^{-}(w) \cap B_{\varepsilon}^{\prime}(y)=\emptyset$ is obtained since $w \in B_{2 \varepsilon_{1}}^{\prime}\left(p_{j}\right) \subset$ $M_{j} \backslash M_{i}$ and $B_{\varepsilon}^{\prime}(y) \subset B_{2 \varepsilon_{1}}^{\prime}\left(p_{i}\right) \subset M_{i}$.

Moreover, we have the following

ClaIM. Under the above notation,

(i) there is $k>0$ such that $g^{-n}(w) \in V_{\varepsilon_{5}}^{\mathrm{s}}\left(p_{j}\right)$ for $0 \leq n \leq k$ and $g^{-k}(w) \in B_{\varepsilon}^{\prime}(z)$,

(ii) there is $m>0$ such that $g^{n}\left(g^{2}(w)\right) \in V_{\varepsilon_{5}}^{\mathrm{u}}\left(p_{i}\right)$ for $0 \leq n \leq m$ and $g^{m}\left(g^{2}(w)\right) \in B_{\varepsilon}^{\prime}(y)$.

Proof. Let us prove assertion (i). First of all, we show $w \notin W_{2 \varepsilon_{1}}^{\mathrm{u}}\left(p_{j}\right)$. If $w \in W_{2 \varepsilon_{1}}^{\mathrm{u}}\left(p_{j}\right)$, then $\mathbf{O}_{g}^{-}(w) \subset W_{2 \varepsilon_{1}}^{\mathrm{u}}\left(p_{j}\right)$. Thus $\mathbf{O}_{g}^{-}(w) \cap B_{\varepsilon}^{\prime}(z)=\emptyset$ by $(18)$. Since $\left(\mathbf{O}_{g}^{+}\left(g^{2}(w)\right) \cup\{g(w)\}\right) \cap B_{\varepsilon}^{\prime}(z)=\emptyset$ (see (19)), we have $\mathbf{O}_{g}(w) \cap B_{\varepsilon}^{\prime}(z)$ $=\emptyset$. This is a contradiction.

Since $w \in B_{\varepsilon}^{\prime}\left(g^{-1}(x)\right) \subset V_{\varepsilon_{5}}^{\mathrm{s}}\left(p_{j}\right)$ (by $\left.(16)\right)$ and $w \notin W_{2 \varepsilon_{1}}^{\mathrm{u}}\left(p_{j}\right)$, there exists $K>0$ such that $g^{-k}(w) \in V_{\varepsilon_{5}}^{\mathrm{s}}\left(p_{j}\right)$ for $0 \leq k \leq K$ and $g^{-K-1}(w) \notin V_{\varepsilon_{5}}^{\mathrm{s}}\left(p_{j}\right)$. Clearly, $g^{-K}(w) \in B_{\varepsilon_{5}}^{\prime}\left(D^{\mathrm{S}}\left(p_{j}\right)\right)$ by Lemma 2.4. Furthermore, $g^{-K-1}(w) \notin$ $B_{\varepsilon_{5}}^{\prime}\left(W_{\varepsilon_{1}}^{\mathrm{s}}\left(p_{j}\right)\right)$. Indeed, since $\mathcal{L}_{g}$ is linear on the neighborhood of $p_{j}$, it is easily checked that

$$
\mathcal{B}_{\varepsilon_{5}}\left(E_{p_{j}, \varepsilon_{1}}^{\mathrm{s}}\right) \subset \bigcup_{k=0}^{\infty} \mathcal{L}_{g}^{k}\left(\mathcal{B}_{\varepsilon_{5}}\left(\varphi\left(D^{\mathrm{s}}\left(p_{j}\right)\right)\right)\right) \cap \mathcal{B}_{2 \varepsilon_{1}}\left(O_{p_{j}}\right) .
$$


Thus

$$
B_{\varepsilon_{5}}^{\prime}\left(W_{\varepsilon_{1}}^{\mathrm{s}}\left(p_{j}\right)\right) \subset \varphi^{-1}\left(\bigcup_{k=0}^{\infty} \mathcal{L}_{g}^{k}\left(\mathcal{B}_{\varepsilon_{5}}\left(\varphi\left(D^{\mathrm{s}}\left(p_{j}\right)\right)\right)\right) \cap \mathcal{B}_{2 \varepsilon_{1}}\left(O_{p_{j}}\right)\right) .
$$

Since

$$
V_{\varepsilon_{5}}^{\mathrm{s}}\left(p_{j}\right)=W_{2 \varepsilon_{1}}^{\mathrm{u}}\left(p_{j}\right) \cup \varphi^{-1}\left(\bigcup_{k=0}^{\infty} \mathcal{L}_{g}^{k}\left(\mathcal{B}_{\varepsilon_{5}}\left(\varphi\left(D^{\mathrm{s}}\left(p_{j}\right)\right)\right)\right) \cap \mathcal{B}_{2 \varepsilon_{1}}\left(O_{p_{j}}\right)\right),
$$

we obtain $g^{-K-1}(w) \notin B_{\varepsilon_{5}}^{\prime}\left(W_{\varepsilon_{1}}^{\mathrm{s}}\left(p_{j}\right)\right)$ (recall $\left.g^{-K-1}(w) \notin V_{\varepsilon_{5}}^{\mathrm{s}}\left(p_{j}\right)\right)$. Hence, by Lemma 2.3(ii), we have $g^{-K^{-}-k}(w) \notin B_{\varepsilon}^{\prime}(z)$ for all $k \geq 0$.

If $g^{-k}(w) \notin B_{\varepsilon}^{\prime}(z)$ for all $0 \leq k \leq K$, then $\mathbf{O}_{g}^{-}(w) \cap B_{\varepsilon}^{\prime}(z)=\emptyset$. Therefore, $\mathbf{O}_{g}(w) \cap B_{\varepsilon}^{\prime}(z)=\emptyset$ since $\left(\mathbf{O}_{g}^{+}\left(g^{2}(w)\right) \cup\{g(w)\}\right) \cap B_{\varepsilon}^{\prime}(z)=\emptyset$ by (19). This is a contradiction.

The other assertion follows from Lemma 2.3(i) and (19) in the same way.

By Claim (i) and Lemma 2.1(ii), we see that

$$
\mathcal{L}_{g}^{-k}(\varphi(w)) \in\left[v_{z}-\varepsilon, v_{z}+\varepsilon\right] \times[-\varepsilon, \varepsilon]
$$

with respect to $E_{p_{j}}^{\mathrm{s}} \times E_{p_{j}}^{\mathrm{u}}$ (recall (12), (13) and (17)). Thus

$\varphi(w)=\mathcal{L}_{g}^{k}\left(\mathcal{L}_{g}^{-k}(\varphi(w))\right) \in\left[\lambda^{k} v_{z}-\lambda^{k} \varepsilon, \lambda^{k} v_{z}+\lambda^{k} \varepsilon\right] \times\left[w_{g^{-1}(x)}-\varepsilon, w_{g^{-1}(x)}+\varepsilon\right]$ (see (10)) and so

$$
\begin{aligned}
\varphi\left(g^{2}(w)\right) & =\mathcal{L}_{g}^{2}(\varphi(w)) \\
\in & {\left[v_{g(x)}-\varepsilon_{6}, v_{g(x)}+\varepsilon_{6}\right] \times\left[c\left(\varepsilon_{1} c^{-1} \lambda^{k}-\varepsilon \lambda^{k}\right), c\left(\varepsilon_{1} c^{-1} \lambda^{k}+\varepsilon \lambda^{k}\right)\right] } \\
& =\left[v_{g(x)}-\varepsilon_{6}, v_{g(x)}+\varepsilon_{6}\right] \times\left[\varepsilon_{1} \gamma^{r k}-c \varepsilon \gamma^{r k}, \varepsilon_{1} \gamma^{r k}+c \varepsilon \gamma^{r k}\right]
\end{aligned}
$$

with respect to $E_{p_{i}}^{\mathrm{s}} \times E_{p_{i}}^{\mathrm{u}}$ by (11) and (16). On the other hand, by Claim (ii) and Lemma 2.1(ii), we have $\mathcal{L}_{g}^{m}\left(\varphi\left(g^{2}(w)\right)\right) \in[-\varepsilon, \varepsilon] \times\left[w_{y}-\varepsilon, w_{y}+\varepsilon\right]$. Thus

$$
\begin{aligned}
\varphi\left(g^{2}(w)\right) & \in\left[v_{g(x)}-\varepsilon_{6}, v_{g(x)}+\varepsilon_{6}\right] \times\left[\varepsilon_{1} \gamma^{1 / 2} \mu^{-m}-\varepsilon \mu^{-m}, \varepsilon_{1} \gamma^{1 / 2} \mu^{-m}+\varepsilon \mu^{-m}\right] \\
& =\left[v_{g(x)}-\varepsilon_{6}, v_{g(x)}+\varepsilon_{6}\right] \times\left[\varepsilon_{1} \gamma^{s m+1 / 2}-\varepsilon \gamma^{s m}, \varepsilon_{1} \gamma^{s m+1 / 2}+\varepsilon \gamma^{s m}\right] .
\end{aligned}
$$

If $r k>s m$, then it is easy to see that $\varepsilon_{1} \gamma^{s m+1 / 2}-\varepsilon \gamma^{s m} \leq \varepsilon_{1} \gamma^{r k}+c \varepsilon \gamma^{r k}$. Hence

$$
\varepsilon_{1}\left(\gamma^{s m+1 / 2}-\gamma^{r k}\right) \leq c \varepsilon\left(\gamma^{r k}+\gamma^{s m}\right)<\left(\gamma^{r k}+\gamma^{s m}\right) \cdot \frac{1}{2} \varepsilon_{1} \gamma^{1 / 2}\left(1-\gamma^{1 / 2}\right) .
$$

Thus

$$
\gamma^{s m+1 / 2}-\gamma^{r k}<\frac{1}{2}\left(\gamma^{r k}+\gamma^{s m}\right) \gamma^{1 / 2}\left(1-\gamma^{1 / 2}\right)
$$

and so $1-\gamma^{r k-s m-1 / 2}<1-\gamma^{1 / 2}$. This is a contradiction since $r k-s m-1 / 2$ $\geq 1 / 2$. 
If $r k \leq s m$, then $\varepsilon_{1} \gamma^{r k}-c \varepsilon \gamma^{r k} \leq \varepsilon_{1} \gamma^{s m+1 / 2}+\varepsilon \gamma^{s m}$. From this, $1-$ $\gamma^{s m-r k+1 / 2}<\gamma^{1 / 2}\left(1-\gamma^{1 / 2}\right)$. This is also a contradiction.

CASE 2: $0<c \leq 1$. Take $\left(0, w_{y}\right) \in E_{p_{i}, \varepsilon_{1}}^{\mathrm{u}}$ and $\left(v_{z}, 0\right) \in E_{p_{j}, \varepsilon_{1}}^{\mathrm{s}}$ such that $w_{y}=\varepsilon_{1} c \gamma^{1 / 2}$ and $v_{z}=\varepsilon_{1}$. Set

$$
y=\varphi^{-1}\left(0, w_{y}\right) \in W_{\varepsilon_{1}}^{\mathrm{u}}\left(p_{i}\right) \quad \text { and } \quad z=\varphi^{-1}\left(v_{z}, 0\right) \in W_{\varepsilon_{1}}^{\mathrm{s}}\left(p_{j}\right),
$$

and fix $0<\varepsilon<\min \left\{\varepsilon_{7},\left(c \varepsilon_{1} / 2\right) \gamma^{1 / 2}\left(1-\gamma^{1 / 2}\right)\right\}$ such that $B_{\varepsilon}^{\prime}(y) \cap W_{2 \varepsilon_{1}}^{\mathrm{s}}\left(p_{i}\right)=\emptyset$ and $B_{\varepsilon}^{\prime}(z) \cap W_{2 \varepsilon_{1}}^{\mathrm{u}}\left(p_{j}\right)=\emptyset$.

Let $0<\varepsilon^{\prime}<\varepsilon$ be as in Case 1, and let $0<\delta=\delta\left(\varepsilon^{\prime}\right)<\varepsilon^{\prime}$ be as in the weak shadowing property of $g$. If we construct a $\delta$-pseudo-orbit $\mathcal{P} \mathcal{O}_{\delta}$ of $g$ in the same way, then there exists a point $w \in B_{\varepsilon}^{\prime}\left(g^{-1}(x)\right)$ weakly $\varepsilon^{\prime}$-shadowing $\mathcal{P O}_{\delta}$. By an analog of the claim in Case 1 (the proof is similar), there are $k, m>0$ such that

$$
\begin{aligned}
\varphi\left(g^{2}(w)\right) \in\left[v_{g(x)}\right. & \left.-\varepsilon_{6}, v_{g(x)}+\varepsilon_{6}\right] \times\left[c\left(\varepsilon_{1} \lambda^{k}-\varepsilon \lambda^{k}\right), c\left(\varepsilon_{1} \lambda^{k}+\varepsilon \lambda^{k}\right)\right] \\
& =\left[v_{g(x)}-\varepsilon_{6}, v_{g(x)}+\varepsilon_{6}\right] \times\left[c \varepsilon_{1} \gamma^{r k}-c \varepsilon \gamma^{r k}, c \varepsilon_{1} \gamma^{r k}+c \varepsilon \gamma^{r k}\right]
\end{aligned}
$$

and

$$
\begin{array}{r}
\varphi\left(g^{2}(w)\right) \in\left[v_{g(x)}-\varepsilon_{6}, v_{g(x)}+\varepsilon_{6}\right] \times\left[c \varepsilon_{1} \gamma^{1 / 2} \mu^{-m}-\varepsilon \mu^{-m}, c \varepsilon_{1} \gamma^{1 / 2} \mu^{-m}+\varepsilon \mu^{-m}\right] \\
=\left[v_{g(x)}-\varepsilon_{6}, v_{g(x)}+\varepsilon_{6}\right] \times\left[c \varepsilon_{1} \gamma^{s m+1 / 2}-\varepsilon \gamma^{s m}, c \varepsilon_{1} \gamma^{s m+1 / 2}+\varepsilon \gamma^{s m}\right]
\end{array}
$$

with respect to $E_{p_{i}}^{\mathrm{s}} \times E_{p_{i}}^{\mathrm{u}}$. If $r k>s m$, then $c \varepsilon_{1} \gamma^{s m+1 / 2}-\varepsilon \gamma^{s m} \leq c \varepsilon_{1} \gamma^{r k}+$ $c \varepsilon \gamma^{r k}$. Since

$$
\varepsilon_{1}\left(\gamma^{s m+1 / 2}-\gamma^{r k}\right) \leq \varepsilon\left(\gamma^{r k}+\frac{1}{c} \gamma^{s m}\right)<\frac{\varepsilon}{c}\left(\gamma^{r k}+\gamma^{s m}\right),
$$

we derive a contradiction. If $r k \leq s m$, then we can also derive a contradiction in the same way.

Suppose that $n_{i}>1$ or $n_{j}>1\left(\right.$ recall $f^{n_{i}}\left(p_{i}\right)=p_{i}$ and $\left.f^{n_{j}}\left(p_{j}\right)=p_{j}\right)$. This case is similar. Indeed, applying Lemma 1.1 (see (1)) to the periodic orbits $\mathbf{O}_{f}\left(p_{i}\right)$ and $\mathbf{O}_{f}\left(p_{j}\right)$, we can construct a diffeomorphism $g\left(C^{1}\right.$-near $f)$ and a system $\mathcal{L}_{g}=\left\{L_{p_{i}}^{n_{i}}, L_{p_{j}}^{n_{j}}, L_{g^{-1}(x)}, L_{x}\right\}$ on the assumption that $x \in$ $g^{-1}\left(W_{\varepsilon_{1} / 2}^{\mathrm{s}}\left(p_{i}\right)\right) \cap g\left(W_{\varepsilon_{1} / 2}^{\mathrm{u}}\left(p_{j}\right)\right)$ is a non-transversal intersection of $W^{\mathrm{s}}\left(p_{i}\right)$ and $W^{\mathrm{u}}\left(p_{j}\right)$ (mimic the procedure used in Step 1). Note that $g$ has the weak shadowing property.

We can prove analogs of Lemmas 2.3 and 2.4 of Step 2 with respect to $\mathbf{O}_{g}\left(p_{k}\right)(k=i, j)$. Since $\varepsilon_{1}$ is sufficiently small, we may assume

$$
\left(g^{n}\left(B_{2 \varepsilon_{1}}\left(p_{k}\right)\right) \cup g^{-n}\left(B_{2 \varepsilon_{1}}\left(p_{k}\right)\right)\right) \cap B_{2 \varepsilon_{1}}\left(p_{k}\right)=\emptyset
$$

for $1 \leq n \leq n_{k}-1$ and $k=i, j$. Choose $y \in W_{\varepsilon_{1}}^{\mathrm{u}}\left(p_{i}\right), z \in W_{\varepsilon_{1}}^{\mathrm{s}}\left(p_{j}\right)$ and $0<\varepsilon^{\prime}<\varepsilon<\varepsilon_{1}$ as in the proof of the simple case, and let $0<\delta=\delta\left(\varepsilon^{\prime}\right)<\varepsilon^{\prime}$ 
be as in the weak shadowing property of $g$. Pick $l>0$ so large that

$$
\max \left\{d\left(g^{-n_{i} l}(y), g^{n_{i} l+1}(x)\right), d\left(g^{-n_{j} l-1}(x), g^{n_{j} l}(z)\right)\right\}<\delta .
$$

Denote by $\mathcal{P} \mathcal{O}_{\delta}$ the following $\delta$-pseudo-orbit of $g$ consisting of three pieces of real $g$-orbits:

$$
\begin{array}{r}
\left\{\ldots, g^{-2}(z), g^{-1}(z), z, g(z), \ldots, g^{n_{j} l-1}(z), g^{-n_{j} l-1}(x), g^{-n_{j} l}(x), \ldots, g^{-1}(x),\right. \\
\left.x, g(x), \ldots, g^{n_{i} l}(x), g^{-n_{i} l}(y), g^{-n_{i} l+1}(y), \ldots, g^{-1}(y), y, g(y), g^{2}(y), \ldots\right\} .
\end{array}
$$

Since $g$ has the weak shadowing property, there exists $w \in B_{\varepsilon}^{\prime}\left(g^{-1}(x)\right)$ weakly $\varepsilon^{\prime}$-shadowing $\mathcal{P} \mathcal{O}_{\delta}$. Clearly,

$$
\begin{aligned}
\mathbf{O}_{g}(w) & =\mathbf{O}_{g}^{+}\left(g^{2}(w)\right) \cup\{g(w)\} \cup \mathbf{O}_{g}^{-}(w) \\
& =\left(\bigcup_{n=0}^{n_{i}-1} g^{n}\left(\mathbf{O}_{g^{n_{i}}}^{+}\left(g^{2}(w)\right)\right)\right) \cup\{g(w)\} \cup\left(\bigcup_{n=0}^{n_{j}-1} g^{-n}\left(\mathbf{O}_{g^{n_{j}}}^{-}(w)\right)\right),
\end{aligned}
$$

where

$$
\mathbf{O}_{g^{n_{i}}}^{+}\left(g^{2}(w)\right)=\left\{g^{n_{i} k}\left(g^{2}(w)\right)\right\}_{k \geq 0} \quad \text { and } \quad \mathbf{O}_{g^{n_{j}}}^{-}(w)=\left\{g^{-n_{j} k}(w)\right\}_{k \geq 0} .
$$

It is easy to see that

$$
\begin{aligned}
& {\left[\mathbf{O}_{g}^{+}\left(g^{2}(w)\right) \cup\{g(w)\} \cup\left(\bigcup_{n=1}^{n_{j}-1} g^{-n}\left(\mathbf{O}_{g^{n_{j}}}^{-}(w)\right)\right)\right] \cap B_{\varepsilon}^{\prime}(z)=\emptyset,} \\
& {\left[\{g(w)\} \cup \mathbf{O}_{g}^{-}(w) \cup\left(\bigcup_{n=1}^{n_{i}-1} g^{n}\left(\mathbf{O}_{g^{n_{i}}}^{+}\left(g^{2}(w)\right)\right)\right)\right] \cap B_{\varepsilon}^{\prime}(y)=\emptyset .}
\end{aligned}
$$

Moreover, assertion (i) (resp. (ii)) of the claim in Step 2 is satisfied by $g^{-n_{j}}$ $\left(\right.$ resp. $\left.g^{n_{i}}\right)$. Thus, applying Plamenevskaya's technique to $L_{p_{k}}^{n_{k}}(k=i, j)$, we get a contradiction.

Acknowledgements. The author wishes to express his deep appreciation to the referee for his careful reading of the manuscript, critical comments and valuable suggestions.

\section{References}

[1] C. Bonatti and L. J. Diaz, Persistent nonhyperbolic transitive diffeomorphisms, Ann. of Math. 143 (1995), 357-396.

[2] J. Franks, Necessary conditions for stability of diffeomorphisms, Trans. Amer. Math. Soc. 158 (1971), 301-308.

[3] S. Hayashi, Diffeomorphisms in $\mathcal{F}^{1}(M)$ satisfy Axiom A, Ergodic Theory Dynam. Systems 12 (1992), 233-253.

[4] M. Hirsch, J. Palis, C. Pugh and M. Shub, Neighborhoods of hyperbolic sets, Invent. Math. 9 (1970), 121-134.

[5] R. Mañé, Contributions to stability conjecture, Topology 17 (1978), 383-396. 
[6] S. Yu. Pilyugin, The Space of Dynamical Systems with the $C^{0}$ Topology, Lecture Notes in Math. 1571, Springer, 1994.

[7] _, Shadowing in Dynamical Systems, Lecture Notes in Math. 1706, Springer, 1999.

[8] O. B. Plamenevskaya, Weak shadowing for two-dimensional diffeomorphisms, Vestnik St. Petersburg Univ. Math. 31 (1998), 49-56 (1999).

[9] C. Robinson, Stability theorems and hyperbolicity in dynamical systems, Rocky Mountain J. Math. 7 (1977), 425-437.

[10] K. Sakai, Pseudo-orbit tracing property and strong transversality of diffeomorphisms on closed manifolds, Osaka J. Math. 31 (1994), 373-386.

[11] —, Shadowing property and transversality condition, in: Dynamical Systems \& Chaos (Tokyo, 1994), Vol. 1, World Sci., Singapore, 1995, 233-238.

[12] -, Diffeomorphisms with the average-shadowing property on 2-dimensional closed manifolds, Rocky Mountain J. Math. 30 (2000), 1129-1137.

[13] S. Smale, The S-stability theorem, Proc. Amer. Math. Soc. 14 (1970), 289-297.

Department of Mathematics

Kanagawa University

Yokohama 221-8686, Japan

E-mail: kazsaka@cc.kanagawa-u.ac.jp sakaik01@kanagawa-u.ac.jp
Current address:

Department of Mathematics

Utsunomiya University 350 Mine-machi Utsunomiya-shi 321-8505, Japan

Received 6 December 1999;

in revised form 12 June 2000 